\title{
Die Magdalenenflut 1342 am Schnittpunkt von Umwelt- und Infrastrukturgeschichte
}

\section{Ein compound event als Taktgeber für mittelalterliche Infrastrukturentwicklung und Daseinsvorsorge}

\author{
Martin Bauch
} St. Mary Magdelene's Flood (1342) at the Intersection of Environmental History and the History of Infrastructu-
res. A Compound Event as a Catalyst of Medieval Infrastructure Development and Public Welfare

\begin{abstract}
This article sheds new light on the disastrous event of St Mary Magdalene's Flood in Central Europe in 1342, which scholars have thus far largely neglected, by examining administrative documents like charters and accounts for the first time, while also considering scientific proxy data like precipitation reconstructions based on tree rings. The result is a much more nuanced reconstruction of these two years that included extreme flooding (in February and July 1342 and July 1343), but also pronounced dryness in spring 1342, which might explain the extreme erosion events geomorphologists attribute to the strong precipitation of these years. The events of 1342/43 are a good example of how a pre-modern, multi-factorial compound event could have disastrous consequences when natural extreme events and disadvantageous socio-economic conditions coincided. Examining a wider variety of written sources reveals that the supra-regional dearth and famine in Central Europe was linked to the fact that the flood occured before grain could be harvested in 1342. Furthermore, the article focuses on infrastructural adaptions like changes in bridge design or large water-infrastructures, as well as on normative reactions at a regional or local level that can be understood, at least partially, to have been caused by the flood disaster. These processes of technological and normative adaption can be understood, on the one hand, as second-order-perceptions of nature in Luhmann's sense; on the other hand, they illustrate the importance of natural extreme events as a catalyst for pre-modern development of infrastruture. In the aftermath of 1342/43, areas to the north of the Alps implemented flood-protection measures to protect the public welfare for the first time, at least such initiatives that went beyond local responses to involve regional and supra-regional powers up to and including the Holy Roman Emperor.
\end{abstract}

Keywords: flooding, St. Mary Magdalene's Flood, infrastructures, criticality, perception of nature, Late Middle Ages

Im vorliegenden Artikel wird das von historischer Seite wenig erforschte Ereignis der Magdalenenflut von 1342 unter Einbeziehung administrativer überlieferung, vor allem aus Urkunden und Rechnungsbüchern, und durch Heranziehung naturwissenschaftlicher Proxydaten, insbesondere aus Baumring-Niederschlagsrekonstruktionen, neu ausgeleuchtet. So gelingt es nicht nur, einen wesentlich differenzierteren verlauf einer insgesamt zweijährigen Hochwasserkatastrophe mit peaks im Februar und Juli 1342, aber auch im Juli 1343 zu gewinnen, sondern auch mehrmonatige Trockenphasen im März bis Juni 1342 erstmals zu fassen, die den (auch in Schriftquellen nachgewiesenen) Erosionseffekt der Starkregenfälle im Juli 1342 besser erklären als bislang. Dabei wird klar, dass 1342/43 ein multifaktorieller compound event vorliegt, der erst durch die Koinzidenz natürlicher Extremereignisse mit sozio-ökonomischen Faktoren zur Katastrophe führt. Die bewusste Ausweitung des Quellenspektrums lässt beispielsweise erstmals eine überregionale Teuerung bei Lebensmitteln im Nachklang der Flutereignisse feststellen, die aber wesentlich im zufälligen Auftreten des Hochwassers noch vor der Getreideernte begründet liegt. Der Fokus des Beitrags liegt auf infrastrukturellen Anpassungsmaßnahmen, etwa veränderten Brückendesigns (am Bsp. der Balduinbrücke in Koblenz) und großer Wasserbaumaßnahmen 
(am Beispiel des Donaudurchstichs von Oberalteich), oder normativen Reaktionen wie den Würzburger Polizeisätzen 1342/43 und der Errichtung von Deichbaugemeinschaften am Niederrhein, die zumindest als indirekte Reaktionen auf die Hochwasserereignisse verstanden werden müssen. Diese technisch-normativen Adaptionsvorgänge können einerseits im Luhmann'schen Sinn als Umweltbeobachtungen zweiter Ordnung verstanden werden, andererseits erläutern sie die Bedeutung von natürlichen Extremereignissen als Taktgeber für vormoderne Infrastrukturentwicklung aufgrund ihrer als kritisch erlebten, ungenügenden Ausgestaltung oder gar ihres völligen Fehlens. Nordalpin sehen wir nach 1342/43 im Bereich des Hochwasserschutzes zaghafte Anfänge obrigkeitlicher Daseinsvorsorge jenseits lokaler Zuständigkeiten.

Schlüsselwörter: Hochwasser, Magdalenenflut, Infrastrukturen, Kritikalität, Naturwahrnehmung, Spätmittelalter

Der Dominikaner Johannes Tauler (†1361), einer der bekanntesten Mystiker und eine Schlüsselfigur der oberrheinischen Gottesfreunde, predigte seit 1320 in seiner Heimatstadt Straßburg vor den ihm anvertrauten Dominikanerinnen, aber auch Pfarreiangehörigen und den Gottesfreunden zuneigenden Laien (Gnädinger 1993: 117-118). In einer Homilie zum Pfingsttag, die über den Einbruch des Heiligen Geistes bei den Jüngern ( $r e-$ pleti sunt omnes spiritu sancto Apg 2,4) reflektierte, verglich der Mystiker, gleich nach der Vorstellung des Schriftwortes und der Begrüßung der Zuhörer, dieses Kommen des Parakleten plastisch mit einem hydrologischen Extremereignis:

Diser minneklicher heiliger geist der kam in die junger und in alle die sin enphenklich warent, mit also grosser richheit und volheit und úberflússikeit und úber gos si inwendig gelicher wise als ob der Rin sin schutz hette und das mittel und hindernisse ab were; wie er denne mit vollem flusse und úber gusse solte komen rúschende, als ob er alles das ertrenken und versenken wolte, und flute alle telre und die grúnde die vor im weren. (Vetter 1910: 304)

Die zitierte Predigt entstammt der maßgeblichen, spätestens 1359 in Köln unter Taulers Aufsicht oder sogar Mitarbeit entstandenen Engelberger Handschrift (Vetter 1910: XIV; Gnädinger 1993: 112-113); jahrgenau datiert ist die Predigt nicht, doch geht die Forschung davon aus, dass sie zwischen 1339 und 1346 aus dem Gedächtnis oder auf der Basis von Notizen niedergeschrieben wurde (Gnädinger 1993: 110). Dabei ist die prominente Rolle, die Johannes Tauler dem Rhein zuschreibt, gar nicht selbstverständlich. Der Alltag in Straßburg wurde von der Ill und ihren Zuflüssen bestimmt (Schenk 2018) und weniger vom in seiner breiten Aue wenige Kilometer östlich der Stadt dahinfließenden Rhein. Warum wählte Tauler also ein Rheinhochwasser zur Veranschaulichung der Geistausgießung? Entscheidend könnte sein, dass er sich in den Jahren 1342/43 gar nicht in seiner Heimatstadt, sondern im Exil in Basel befunden hatte. Dort lebte 
er mit seinen dominikanischen Mitbrüdern im Predigerkonvent, direkt am Rheinufer gelegen (Gnädinger 1993: 33-43).

Ist es also möglich, dass der oberrheinische Mystiker auf eine autobiographische Erfahrung mit einem bekannten meteorologischen Extremereignis des Spätmittelalters rekurriert, die sogenannte Magdalenenflut von 1342, die auch gerne als „Jahrtausendflut/millennium flood“ (Tetzlaff et al. 2002; Bork et al. 2011; Herget et al. 2015) oder „hydrologischer Gau“ (Zbinden 2011) bezeichnet wird? Tatsächlich betraf das Hochwasserereignis ein ausgesprochen großes Gebiet, von der Rhone bis nach Ungarn (Kiss 2009), von den Alpen bis nach Norddeutschland, und ihre Spuren sind in historischen Dokumenten ebenso zu finden wie in Baumringen, Sedimentbohrkernen und geomorphologischen Schnitten von Schwemmfächern. Umso erstaunlicher ist es, dass keine umfassende Studie des Ereignisses von historischer Seite vorliegt, wenn es auch erste Ansätze für lokale Zugriffe (Gauger 2010) oder grobe Überblicke (Rohr 2007: 226-228; Bauch 2014) gibt. Die narrativen Quellen zum Ereignis liegen bereit (in unkritischer Auswahl bei Weikinn 1958: 197-223; verlässlicher bei Alexandre 1987: 467-470) und auch die wenigen zeitgenössischen Inschriften sind aufgearbeitet, so für Hannoversch-Münden (Wehking 2006) und Göttingen (Arnold 1980; Bauch 2016). Nicht systematisch untersucht wurde bisher die administrative Überlieferung der Jahre 1340-1345; gemeint sind damit vor allem über 300 Urkundenbücher, aber auch normative und fiskalische Quellen aus dem ganzen oben skizzierten Raum, die vom Autor fast ausnahmlos in edierter Form ausgewertet wurden.

Als meteorologische Ursache der Flut ist verschiedentlich eine Vb-Tiefdruckbahn ins Spiel gebracht worden (Jacobeit et al. 2006), wie sie auch für die Hochwasser 1997, 2002 und 2013 in Mitteleuropa verantwortlich war. Andere Vorschläge deuten auf eine mitteleuropäische Troglage (Herget et al. 2015), doch zweifellos trifft zu, dass noch mehr „indications about circulation dynamics on a sub-monthly scale“ (Jacobeit et al. 2006: 961) notwendig sind, um mit einer soliden meteorologischen Erklärung des Ereignisses aufzuwarten. Hydrologen haben auf der Basis von historischen Dokumenten das Flutereignis vom Juli 1342 untersucht und schätzen einen Hochwasserpegel vom 19. bis 25. des Monats in Frankfurt von 7,85 m über normal, was den absoluten historischen Rekordpegel definiert; an der Mündung der Tauber sind 8,5 m errechnet worden (Tetzlaff et al. 2002). Der Abfluss an der Mündung des Main in den Rhein soll $3500 \mathrm{~m}^{3} / \mathrm{s}$ betragen haben, ungefähr die doppelte bis dreifache Menge, die von Hochwasserereignissen des 20. Jahrhunderts bekannt ist. Der Kalkulation der Hydrologen zufolge müsste der Abfluss des Hochwassers vier Wochen gedauert haben, bei einer kalkulierten Niederschlagsmenge von $1751 / \mathrm{m}^{2}$ in nur vier Tagen (ebd.: 42-48). Bei all diesen Schätzungen ist aber zu beachten, dass sie auf 
Angaben zu einer einzigen Hochwassermarke in der Frankfurter Weißfrauenkirche zurückgehen, die 1944 zerstört wurde und von der nicht gesagt werden kann, ob sie verlässlich den Wasserstand des Jahres 1342 anzeigte oder in 600 Jahren nicht doch verschiedentlich versetzt wurde.

Damit ist das prekäre Verhältnis von historischer Überlieferung und naturwissenschaftlicher Forschung zum Thema bereits angerissen. Eine ähnlich fundamentale, manchmal fragile Rolle spielt die historische Überlieferung bei der Feindatierung historischer Erosionsereignisse.

Dabei bietet sich gerade ein Extremereignis wie die Magdalenenflut an, das eindeutige Spuren in natürlichen wie gesellschaftlichen Archiven hinterlassen haben müsste, den seit einigen Jahren von der Klimageschichte propagierten consiliant approach to history (McCormick 2011; Izdebski 2016) für eine Periode mit reichlich greifbarer Quellenüberlieferung zu testen. Ausführlicher wurde dieser Ansatz im Forum Genetic History II des vorangegangenen Bandes dieser Zeitschrift besprochen (Bauch 2019). Darüber hinaus ist zu klären, ob wir das Flutereignis wirklich getrennt von anderen Extremereignissen und natürlich sozio-ökonomischen Rahmenbedingungen betrachten können. Vielleicht ist es sinnvoll, die Magdalenenflut und ihren Impact als compound event zu beschreiben, also als „the combination of multiple drivers and/or hazards that contributes to societal or environmental risk" (Zscheichler et al. 2018: 470). Für den dort vorgeschlagenen Ansatz ist ein bottom-up approach typisch, der alle relevanten Elemente identifizieren soll, die zu einer besonders großen Schadenswirkung beitragen könnten. Der Einbezug bisher wenig beachteter administrativer Überlieferung und regionalisierter Proxydaten bietet sich für ein solches Vorgehen an.

Nicht zuletzt legt das angeführte Predigtzitat Taulers die Frage nahe, inwiefern das Ereignis von 1342 Einzug ins Umweltwissen der Zeitgenossen gefunden hat. Eine Interaktion von Extremereignis und gesellschaftlichen Vorstellungen über die natürliche Umwelt finden wir eindrücklich auch in der Mitte des 14. Jahrunderts, so etwa beim Erdbeben von Villach im Januar 1348 (Rohr 2007: 131-166), das Konrad von Megenberg in seinen Traktaten ausführlich behandelt, etwa im „Buch der Natur“ (Luff \& Steer 2003) oder natürlich noch viel deutlicher bei der umfangreichen theologischenastrometeorologischen Theoriebildung zum biologischen Epochenereignis des Schwarzen Todes (Horrox 1994). Der schon erwähnte Konrad von Megenberg sieht sogar, ausgehend von aristotelischen Ansätzen, einen Zusammenhang zwischen Erdbeben und dem nachgelagerten Auftreten der Pest (Krüger 1972). Da aber ein in jeder Hinsicht herausragendes Ereignis wie die Magdalenenflut keine größeren Spuren in den naturkundlichen Traktaten der Zeit hinterlassen hat, und nur peripher in anderen kulturellen Bereichen reflektiert wurde - etwa, wie angedeutet, in wenigen Inschrif- 
ten oder der einleitend angeführten Tauler-Predigt -, muss nach Formen eines „Umweltwissens zweiter Ordnung“ gefragt werden: Indirekten Reflexionen über das Ereignis und seinen sozio-ökonomischen Impact, also im Luhmann'schen Sinn Beobachtungen der historischen (Natur-)Beobachtungen (Gumbrecht 2013), die aber nicht fragen „Was passiert?", sondern das „Wie?" von Hochwasserschäden und Nahrungsmängel ausleuchten und so den primär straftheologischen Deutungshorizont überschreiten können. Das bedeutet etwa, dass Adaptionen im normativen und infrastrukturellen Bereich untersucht werden, die keine direkten Bezüge auf die Naturkatastrophe aufweisen, die aber ohne das Flutereignis und seine direkte Beschreibung durch Chronisten und andere Zeitgenossen in ihrer Charakteristik und Chronologie nur schwer zu erklären sind.

Der vorliegene Beitrag verfolgt daher drei Ziele:

1. Die Gewinnung von neuen Details über den Verlauf, vor allem aber den sozio-ökonomischen Impact der Magdalenenflut aus der bisher kaum herangezogenen, nicht-narrativen Überlieferung, insbesondere was Versorgungskrisen und regulative Adaption angeht.

2. Die Verschränkung historischer Quellen mit naturwissenschaftlichen Daten, wo immer sich diese ergänzen oder korrigieren lassen; und das nicht zuletzt mit dem Ziel, andere natürliche Extremereignisse zu bestimmen, die bisher im Schatten der Juliflut von 1342 gestanden haben.

3. Die Relevanz von Extremereignissen für die vormoderne Infrastrukturgeschichte zu diskutieren und an dem Fall der Magdalenenflut gleichsam exemplarisch zu erproben, ob erste Ansätze mittelalterlicher Daseinsvorsorge ursächlich auf die singuläre Hochwassererfahrung zurückgeführt werden können.

Somit überschneiden sich in diesem Beitrag die Erkenntnisinteressen der disaster history, der Klimageschichte und der relativ neuen, vormodernen Infrastrukturgeschichte (Engels \& Schenk 2014). Insbesondere letztere ist noch erläuterungsbedürftig: Den neuzeitlichen Begriff der Infrastruktur (Laak 1999; Engels \& Obertreis 2007) samt zugehöriger Konzepte auf das Mittelalter anzuwenden, ist nicht selbstverständlich (Schenk \& Eifert 2018: 49-54). Aber er kann in Form eines „kontrollierten Anachronismus“ (von Moos 1998) fruchtbar gemacht werden. Von der Quellenlage begünstigt sind für das Mittelalter Wasserinfrastrukturen, zu denen bereits etliche Studien vorliegen (Förster \& Bauch 2015; Bütow 2015; Huber-Rebenich et al. 2017). Mittelalterliche Infrastrukturen im Allgemeinen sind dabei jüngst in einem programmatischen Beitrag in drei Teilbereiche gegliedert worden (Schenk \& Eifert 2018: 53): 1. Die materielle, feste oder bewegliche Struktur, 2. Soziokulturelle Praktiken (und Trägergruppen) sowie 3. Spezi- 
fische Ordnungskonfigurationen, das heißt Deutungen und Vorstellungen von Infrastruktur.

Diese in sich logische Dreiteilung von Infrastrukturen, verstanden als „netzwerkartige, komplexe Systeme, die wegen ihrer materiellen Persistenz Konstellationen und Entscheidungen der Vergangenheit gleichsam speichern, Eigendynamiken entwickeln und dadurch Macht auf die jeweilige Gegenwart ausüben" (ebd.: 51), legt den Schwerpunkt auf die Geschichte bereits existierender Infrastrukturen und ihre Neubewertung im Krisenfall (Engels \& Nordmann 2018). Demgegenüber wurde die Genese von Infrastrukturen, der spezifische historische Moment und damit die ausschlaggebenden Faktoren für die Schaffung einer begrenzt oder substantiell neuen Infrastruktur für das Mittelalter bisher weniger beachtet. Ein natürliches Extremereignis wie die Magdalenenflut, das einerseits viele existierende Infrastrukturen beschädigte oder zerstörte, und andererseits eine potentiell traumatische Verunsicherung der spätmittelalterlichen Gesellschaften darstellte, lädt dazu ein, den Blick auf die Entwicklung von Infrastrukturen nach einem großen externen Schock zu werfen. Gewissermaßen verfestigt sich das Umweltwissen zweiter Ordnung über eine spezifische Naturkatastrophe in den auf lange Dauer angelegten Wasserinfrastrukturen, die nach dem Ereignis errichtet werden. In dieser auf die Jahre 1342-1345 beschränkten Momentaufnahme als Post-Desaster-Variante der SchenkEifert'schen Kritikalitätszumessung kann hoffentlich die angestrebte „Spezifität von Krisenphänomenen in den Quellen fassbar [gemacht und auch ein] Ansatz zur Untersuchung der Bedeutung, der Funktion und des Wandels von Infrastrukturen" (Schenk \& Eifert 2018: 48) geliefert werden.

Da die Auswahl einer historischen Fallstudie (Pohlig 2013) begründet werden muss, erst recht, wenn sie möglicherweise paradigmatisch (Meier 2016) sein könnte, sei noch einmal auf die oben erwähnten Forschungspositionen verwiesen: Die Flutereignisse von 1342 gelten als singulär im zweiten Jahrtausend, was Intensität und geographische Ausdehnung angeht. Sie sind sowohl in den Archiven der Natur wie in den Archiven der Gesellschaft in hoher Dichte und großer zeitlicher Auflösung überliefert. Außerdem ist die Magdalenenflut als Großereignis wenige Jahre vor dem Auftreten des Schwarzen Todes als demographisch-epidemologischem Dreh- und Angelpunkt der europäischen Geschichte zwischen 1000 und 1800 von besonderem Interesse für die sogenannte „Krise des Spätmittelalters". Von klimahistorischer Seite ist wiederum betont worden, dass die gesamten 1340er Jahre Ausnahmecharakter hatten: Die magistrale Synthese Bruce Campbells von Wirtschafts-, Klima- und Epidemiegeschichte (Campbell 2016) betont den Übergangscharakter der Dekade; sie sei der tipping point der sogenannten "Great Transition“, des Übergangs von der Mittelalterlichen Klimaanomalie zur Kleinen Eiszeit (ebd.: 283-283; 
zu den Perioden White 2014), der von einer starken Varianz ganz unterschiedlicher, klimatischer Proxydaten gekennzeichnet ist (Campbell 2016: 267-331). Tatsächlich ergibt sich aus unabhängigen paläoklimatologischen Daten, dass die 1340er und die frühen 1350er Jahre einen Zeitabschnitt fast einzigartiger klimatischer Instabilität darstellten. Einerseits weisen Temperaturrekonstruktionen der Nordhemisphäre die Jahre 1344-53 als siebtkältestes Jahrzehnt der letzten 2.500 Jahre aus (Sigl et al. 2015, Fig. 3 und Extended Data Table 5), andererseits zeigen aggregierte Daten zum Baumwachstum, dass sich in den Jahren 1341 bis 1354 alle Chronologien von Dendrodaten aus beiden Hemisphären gleichförmig in Richtung schmalerer Jahresringe bewegen, mit einem kollektiven Wachstumsminimum in den Jahren 1348-1350 (Campbell 2016: 285 und Fig. 4.2.; Sigl et al. 2015, Extended Data Table 5). Eine ganz andere Nutzung von Baumringdaten hat vor kurzem Frederik Charpentier Ljungqvist vorgelegt, wobei er für Westund Mitteleuropa über mehrere hundert Jahre die aggregierten Fälldaten von archäologisch untersuchtem Holz quantitativ ausgewertet hat. Dabei verfolgt er die Hypothese, dass diese meist in Gebäuden verbauten Hölzer rein quantitativ gesellschaftliche Bautätigkeit widerspiegeln und daher zum Beispiel bei Pestwellen ein numerischer Rückgang dieses präzise datierbaren Bauholzes festzustellen sein müsste. $\mathrm{Zu}$ seiner Überraschung lagen Tiefpunkte der Bauaktivität auch schon vor dem Auftreten der Pest in Europa, und eine Phase prononcierten Rückgangs findet sich schon zu Anfang der 1340er Jahre (Charpentier Ljungqvist et al. 2018, Fig. 3A). Konkret ist zwar das Pestjahr 1349 das Jahr mit den absolut geringsten Fällzahlen im ganzen Erfassungszeitraum 1250-1700, doch auf Platz 2 und 3 folgen die Jahre 1340 und 1344 (ebd.: 82) - ein Grund mehr, sich die Magdalenenflut 1342 und ihr zeitliches Umfeld noch einmal genauer vorzunehmen.

Im Folgenden wird in zwei größeren Abschnitten dargestellt, was sich mit der deutlich erweiterten Quellenbasis aus Proxydaten einerseits und administrativ-fiskalischen Dokumenten andererseits über den Impact der untersuchten Extremereignisse auf bestehende Infrastrukturen und die Nahrungsmittelsicherheit erkennen lässt. In einem zweiten Schritt geht es um Neubau/Adaptionen von Infrastrukturen und die Anpassung von Normen in Reaktion auf die sozio-ökonomischen Veränderungen nach 1342. Abschließend soll diskutiert werden, ob die aufgeworfenen Fragen nun besser beantwortbar sind als zuvor. 


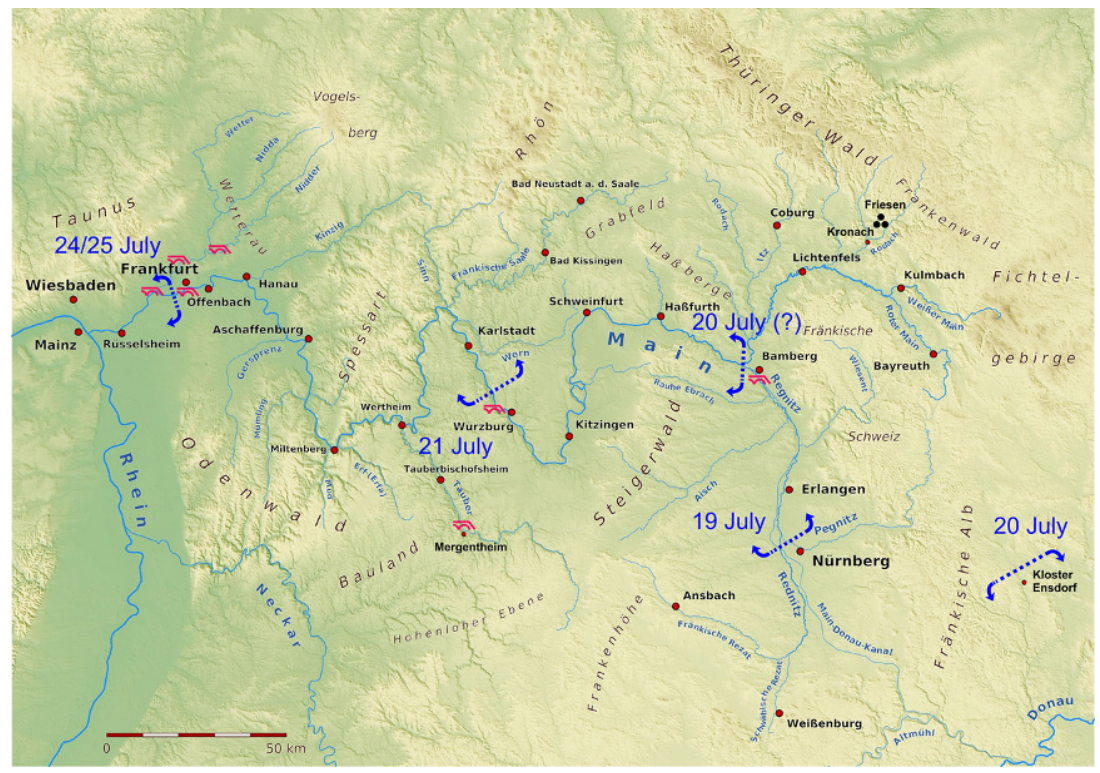

Abb. 1 Rekonstruktion des wandernden Hochwasserscheitels der Juliflut 1342 im Einzugsgebiet des Main. (Karte: BerndH/Modifikationen Martin Bauch, Quelle: Wikimedia Commons: https://de.wikipedia.org/wiki/Main\#/media/File:Main-Karte-160710.jpg CC BY-SA 3.0)

\section{Impact der Extremereignisse von 1342/43}

\section{Ergänzungen des ereignisgeschichtlichen Ablaufs}

Es ist aus der bisherigen Forschung lange bekannt, dass eine erste Hochwasserwelle im Februar 1342 vor allem die Elbe und Donau betraf und dass es der Eisstau war, der nach aller Plausibilität die größten Schäden an den bestehenden Brücken anrichtete. Die zweite Hochwasserwelle im Juli des Jahres war wohl an Main, Rhein, Weser und Donau am stärksten die Bezeichnung als Magdalenenflut im Hinblick auf einen mutmaßlichen Höhepunkt der Flut um den 22. Juli herum ist tendenziell irreführend und doch ein etablierter Begriff, an dem aus pragmatischen Gründen festgehalten werden soll. Der Scheitelpunkt der Flutwelle erreichte selbst Frankfurt erst in der Nacht vom 24. auf den 25. Juli (Abb. 1), so dass der Magdalenentag wohl in der Memoria der Flut, nicht aber in ihrem tatsächlichen Ablauf eine herausragende Rolle spielte.

Nur partiell lassen sich die bereits von Curt Weikinn gesammelten Belege (Weikinn 1958: 197-237) über die Ausdehnung des von der Flut betroffenen Gebiets noch ergänzen: So ist etwa der Unterlauf der Weser (Bork \& Bellstedt 2000: 570) ins Blickfeld geraten, und von der Fulda gibt es etwas unklare Befunde zur Überflutung der Neustadt von Kassel (Alex- 
andre 1987: 468). Dass auch die Leine als Nebenfluss der Weser betroffen gewesen sein dürfte, ergibt sich aus der auf die Magdalenenflut Bezug nehmenden Inschrift eines Reliquienkreuzes aus der Göttinger Kirche St. Alban (Bauch 2016). Hochwasserberichte im Juli 1343 vom Hoch- und Oberrhein und seinen Zubringern (Weikinn 1958: 217-219), die schnell im Verdacht stehen, als Doppelungen des 1342er Ereignisses in späterer oder zeitgenössischer Überlieferung mitgeschleppt worden zu sein, lassen sich erstmals durch eine Chronologie der Wasserstände vom Niederrhein verifizieren. Die Hofrechnungen des Herzogs von Geldern, die Werner von Deventer verfasst hat, überliefern bereits für Anfang Juli 1342 mindestens einen drastischen Anstieg des Wasserstands. Am Ende des Monats hielt das Hochwasser an, so dass der herzögliche Rechnungsführer noch am 2. August von Knechten durch die überfluteten Gebiete begleitet werden musste. Am 8. und erneut am 12. August musste der Herzog selbst mit seinem Gefolge auf Schiffen über die überflutete Betuwe transportiert werden. Die Wasser scheinen sich nicht so schnell zurückgezogen zu haben, denn noch im Januar und Februar 1343 wird das "große Wasser" als Realität beschrieben, und das Julihochwasser 1343 - das die Berichte vom Oberlauf des Stroms verifiziert - ließ sich noch Anfang August am Niederrhein beobachten, als Boten des Herzogs die Wasserstände prüften (Janssen 1970: 234). Auch die dendrochronologischen Daten für den Niederrhein (Abb. 2a) bestätigen, dass $1342 \mathrm{zu}$ den zehn feuchtesten Jahren des Jahrhunderts gehörte, während 1343 nur wenig herausragt.

Weniger Beachtung hat gefunden, dass es im Frühjahr 1342 und bis in den Juli hinein eine ausgeprägte Trockenheit in Teilen Mitteleuropas gab: Explizit erwähnen sie Chroniken aus Westfalen und Hessen (Weikinn 1958: 215-216). Auch das berühmte Wettertagebuch des William Merle, der 1337 bis 1344 um Oxford herum seine Beobachtungen notierte, erwähnt die Abwesenheit von Regen in diesen Monaten (Symons 1891: 24). Die Baumringrekonstruktionen des Old World Drought Atlas (OWDA) (Cook et al. 2015) für die Vegetationsperiode der Jahre 1336-45 zeigen für Mitteleuropa in der Tat zwischen 1336 und 1341 eher trockene Sommermonate, wenn auch keine extreme Dürre; für 1342/43 ist der Befund dann eher feucht, wenn auch nicht so ausgeprägt nass, wie man erwarten würde (Abb. 3). Auch die Rekonstruktion eines Durchschnittswerts für Mitteleuropa auf Basis der OWDA-Daten für Niederschläge im Zeitraum Juli-August (Abb. 3) zeigt 1342/43 zwar als zu den zehn feuchtesten Sommern des 14. Jahrhunderts gehörig, aber keineswegs als singulär nass. $\mathrm{Zu}$ diesen Befunden gesellt sich die Ballung von vier zweifelsfrei belegten Stadtbränden im Reich nördlich der Alpen in wenigen Monaten: Am 31. März brannte halb Eisenach nieder (Weigelt 2007: 83), am 8. Mai 1342 Breslau (Mühle 2015: 106; Büsching 1813: 115) und am 5. Juni Lim- 


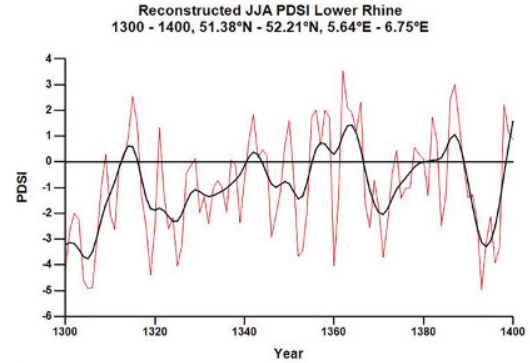

a

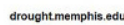

Reconstructed JJA PDSI Mosel Area $1300-1400,49.71^{\circ} \mathrm{N}-50.46^{\circ} \mathrm{N}, 6.48^{\circ} \mathrm{E}-7.77^{\circ} \mathrm{E}$

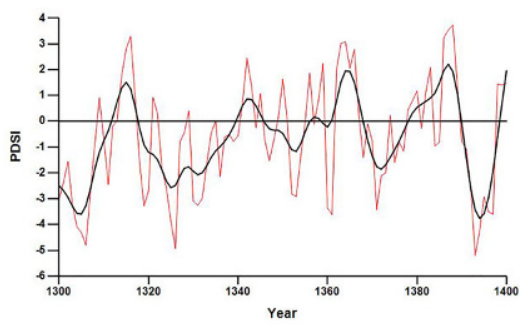

C

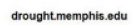
Reconstructed JJA PDSI Franconia

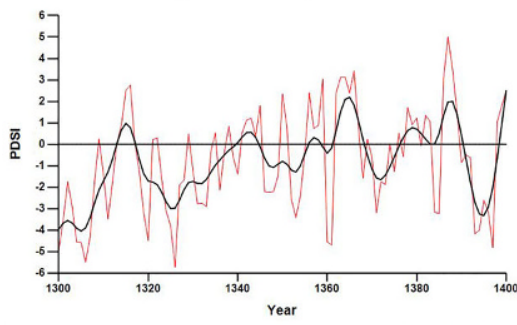

Reconstructed JJA PDSI Central Neckar Valley
$1300-1400,48.41^{\circ} \mathrm{N}-48.88^{\circ} \mathrm{N}, 8.72^{\circ} \mathrm{E}-9.83^{\circ} \mathrm{E}$

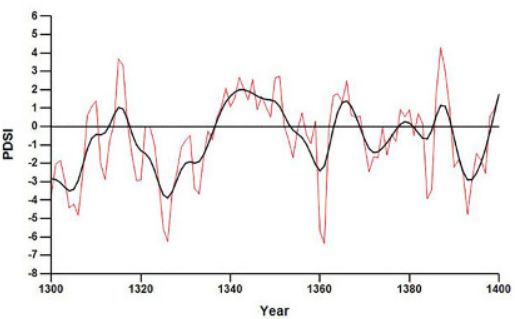

b

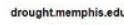
Reconstructed JJA PDSI Bohemia
$1300-1400,48.75^{\circ} \mathrm{N}-50.66^{\circ} \mathrm{N}, 13.1^{\circ} \mathrm{E}-16.35^{\circ} \mathrm{E}$

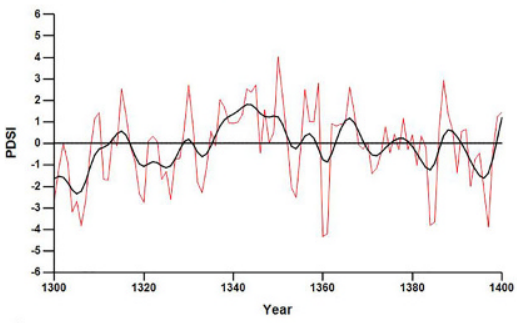

d Reconstructed JJA PDSI Lower Bavaria
(3)

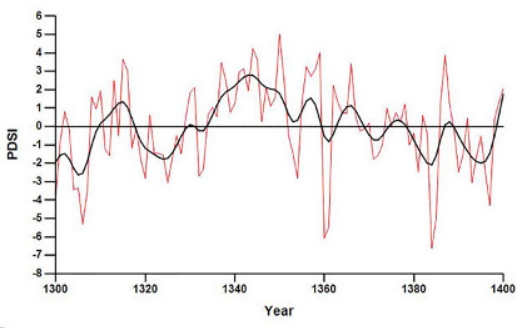

f

Abb. 2 Jahrgenau aufgelöste Rekonstruktion des selbst-kalibrierenden Palmer Drought Severity Index (PDSI) für (a) den Niederrhein, (b) den Mittleren Neckar, (c) die Mosel zwischen Trier und Koblenz, (d) Böhmen, (e) Franken zwischen Bamberg und Würzburg und (f) Niederbayern zwischen Regensburg und Passau, jeweils mit Bezug auf die Sommerniederschläge zwischen Juni und August im Lauf des gesamten 14. Jahrhunderts. (Daten: Old World Drought Atlas (OWDA), vgl. Cook et al. 2015 und http:// drought.memphis.edu/owda)

burg (Wyss 1883: 28). Am bekanntesten ist zweifellos der Stadtbrand von Landshut am 6. Mai 1342, dem 112 Häuser zum Opfer fielen, als starke Winde jeden Löschversuch zunichte machten (Herzog 1963: 263). Ein nicht näher beschriebener Stadtbrand vor 1343 war unter anderen ein Argument für die Verlegung der Stadt Benatek in Nordböhmen (Haas 1954: 76; Tadra 1880: 510-512). Hinsichtlich der Frühen Neuzeit ist überzeugend argumentiert worden, dass Trockenperioden der zweite große Risikofaktor 

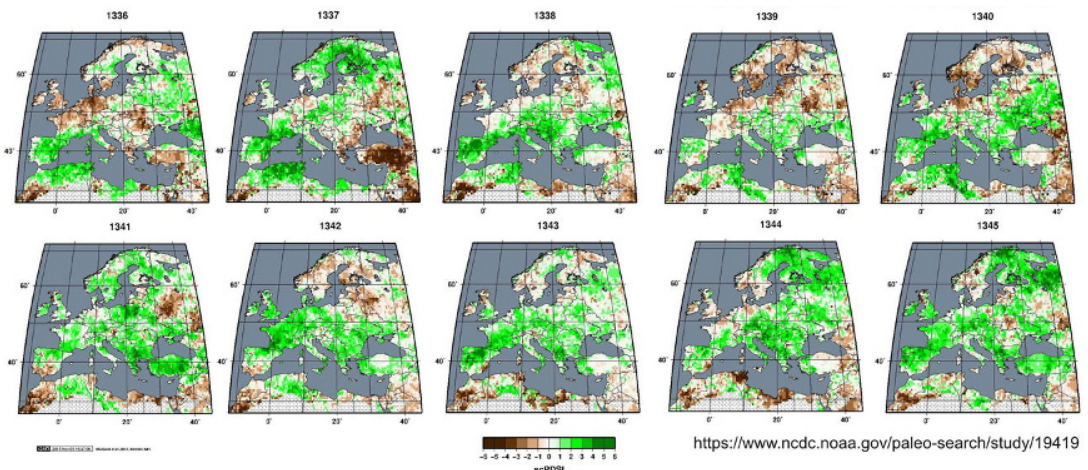

Reconstructed JJA PDSI Central Europe $1300-1400,46.81^{\circ} \mathrm{N}-54.54^{\circ} \mathrm{N}, 4.88^{\circ} \mathrm{E}-19.45^{\circ} \mathrm{E}$

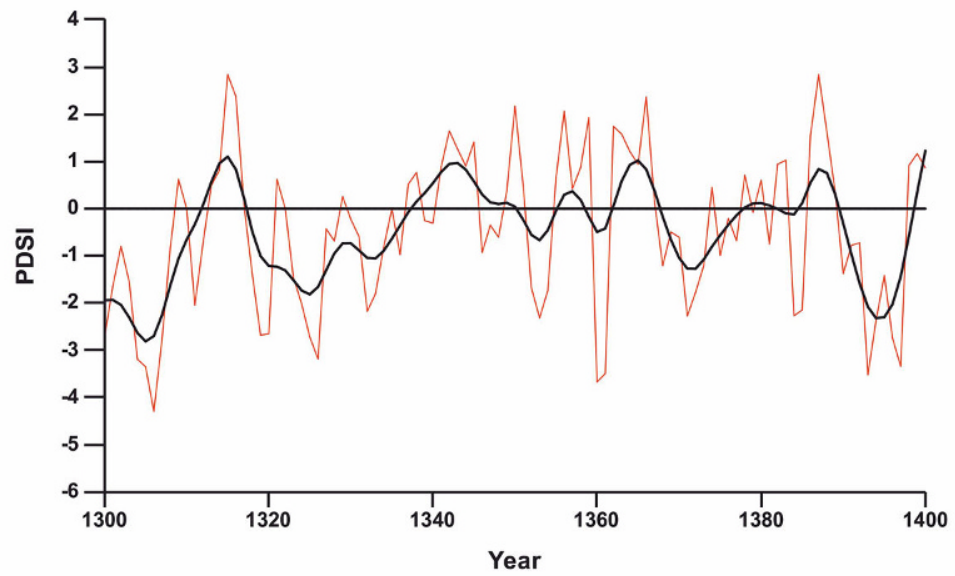

drought.memphis.edu

Abb. 3 Oben: Räumlich aufgelöste Rekonstruktionen der Niederschläge von Juli bis August in den Jahren 1336-45 auf der Basis von Baumringdaten und wiedergegeben mit Werten des selbstkalibrierenden Palmer Drought Severity Index (PDSI),von maximaler Feuchtigkeit +6 bis maximaler Trockenheit -6 . Dabei dienen 106 Baumringchronologien als Datengrundlage für die berechnete Rekonstruktion der lokalen Feuchtigkeitsverhältnisse an über 5400 Netzpunkten, die über den gewählten Kartenausschnitt Europas, des Nahen Ostens und Nordafrikas gelegt wurden. Die Verfügbarkeit von bis ins 14. Jahrhundert vorliegenden Baumringchronologien und damit die Verlässlichkeit der Rekonstruktion für Mitteleuropa ist besser als für die meisten anderen Regionen in der Untersuchung. (Daten und Karten: Cook et al. 2015; genauer Einblicke in Methode und Datengrundlage in den Supplementary Materials https://advances.sciencemag.org/cgi/ content/full/1/10/e1500561/DC1). Unten: Jahrgenau aufgelöste Rekonstruktion des selbst-kalibrierenden Palmer Drought Severity Index (PDSI) für Mitteleuropa mit Bezug auf die Sommerniederschläge zwischen Juni und August im Lauf des gesamten 14. Jahrhunderts. (Daten: Old World Drought Atlas (OWDA), vgl. Cook et al. 2015 und http:// drought.memphis.edu/owda) 
neben Kriegsereignissen für Brandkatastrophen in vormodernen Städten waren (Zwierlein 2011).

Diese kurzen Ergänzungen des ereignisgeschichtlichen Ablaufs zeigen schon ein deutlich differenzierteres Bild: Das Jahr 1342 war keineswegs ausschließlich von Hochwasser oder kontinuierlichem Niederschlag geprägt - vielmehr wechselten die Extreme vom schneereichen und kalten Winter 1341/42 zum frühen Tauwetter im Februar des Jahres samt Schneeschmelze und Eisstößen, dann lang anhaltende Trockenheit mit Begünstigungen von Stadtbränden. Dem folgten die extremen Niederschläge im Juli, die dann - wie zumindest für den Niederrhein gezeigt werden konnte - langanhaltende Überschwemmungen auslösten. Im Folgenden soll daher der Begriff der Magdalenenflut im Bewusstsein verwendet werden, dass wir es tatsächlich mit mehreren Flutereignissen der Jahre 1342/43 zu tun haben, die zudem um eine ausgeprägte Phase der Trockenheit im Frühjahr/ Frühsommer 1342 zu ergänzen sind.

Wasserinfrastrukturen: Brücken und Mühlen

Bereits bekannt war, dass im Februar 1342 und im Juli des Jahres die Mainbrücken in Frankfurt, Würzburg, Bamberg sowie die Donaubrücke in Regensburg zerstört oder schwer beschädigt worden waren. Dasselbe gilt für die Brücken über die Elbe in Dresden und Meißen sowie über die Weser in Minden und die Querungen kleinerer Flüsse in Mitteldeutschland wie der Gera im Fall von Erfurt, der Unstrut für Wetzendorf und der Werra für Vacha, Meiningen und Eisenach (Weikinn 1958: 199-201, 205-215). Hinzu kamen zerstörte Brücken über die Lahn in Weilburg (Deinhard 1988: 234) und Marburg (Klein 2011: 264), und im selben Jahr 1342 wurde die Spreebrücke in Bautzen weggespült (Liebescher \& Schmiedel 1999: 262). Jüngst wurde auf die Hochwasserwelle 1343 im Südwesten des Reichs hingewiesen, die entlang des Hoch- und Oberrheins und seiner Zubringer die Brücken in Zürich, Säckingen, Rheinfelden, Basel und Laufenburg zerstörte (Camenisch 2019).

Doch während diese Hinweise alle aus narrativen Quellen stammen, finden sich weitere Indizien in Urkunden, die Informationen über Reparaturbemühungen geben: So unterstützte Kaiser Ludwig IV., genannt der Bayer, durch Zollvergaben im September 1342 den Aufbau der Frankfurter Mainbrücke, aber auch die Rekonstruktion von vier Brücken im Umland der Mainmetropole (Böhmer 1836: 578-579). Ähnlich handelte er $1343 \mathrm{im}$ Fall von Schwäbisch Hall (Knöpfler 1902: 348). Ob er mit der Abgabe all seiner Brückenrechte an die Stadt Ingolstadt im April 1342 - unter Auflage der Instandhaltung (Hübner 1803: 77) - auf bereits vorhandene Schäden reagierte, ist unklar. Es ist ein methodisch kaum lösbares Problem, aus Reparaturbemühungen wenige Jahre nach einem (lokal nicht explizit be- 
legten) Hochwasserereignis auf diese Flut rückzuschließen. So wissen wir um umfangreiche Brückenreparaturen in Zittau im Jahr 1345 (Emler 1892: 627) oder 1342 um ein Testament zugunsten der Wiederherstellung einer Egerbrücke in Louny (Müller 1929: 9), während 1346 mit dem Bau einer Tauberbrücke in Mergentheim begonnen wurde (Kolbe 1988: 42), wobei nicht klar ist, ob hier eine Vorgängerin existierte. Während wir also im Fall von Dresden, wo die Elbbrücke ab 1344 in Stein neu erbaut wurde (Pfeiffer \& Schmiedel 1999: 194-195) und bei der Mainbrücke in Frankfurt, deren neuer südlicher Turm bereits 1348 vollendet war (Wisselbach 2010: 27), um die vorangegangen Flutschäden wissen, lassen sich andere Erwähnungen von Brückenreparaturen/-neubauten nur mit gewisser Plausibilität in den Kontext eines Extremflutereignisses einordnen. Generell fehlt eine quellenkritisch arbeitende Altbrückenforschung für Mitteleuropa, um den Schaden der Hochwasserjahre 1342/43 verlässlich abschätzen zu können. Dass ein solches Unterfangen lohnen dürfte, kann die mitteleuropäische Überblickskarte nur für diese Jahre zu Brückenschäden und -neubauten zeigen (Abb. 4).

Wenn wir Wasserinfrastrukturen jenseits von Brücken ins Auge fassen, erwähnen narrative Quellen zwar regelmäßig Schäden an Dämmen, Wehren und Wassermühlen (etwa im Februar 1342 in Prag, Zachová 1998: 178-179). Doch in Urkunden ist der Nachweis solcher Effekte schwierig: Mühlenkaufverträge scheinen Schäden eher zu verschweigen (Bode 1905: 130-131), während die Dokumentation von Streitigkeiten um die Mühlennutzung gelegentlich auf Hochwasserschäden (Verein für mecklenburgische Geschichte und Althertumskunde 1875: 478) und deren - künftige oder bereits eingetretene - Reparatur verweist (ebd.: 613-614). Spuren der Fluten können auch vermutet werden, als 1345 in Brandenburg ein Straßendamm über die Havel repariert werden musste (Riedel 1869: I 9, 39), und schon 1343 trat Ludwig der Bayer an die Bürger seiner Stadt Landshut Zölle ab unter der Auflage, dass sie das Donauwehr reparieren sollten (Herzog 1963: 264-265). Dass für dessen schlechten Zustand der Stadtbrand des Vorjahres ursächlich war, scheint wenig plausibel. Die Beschädigung von Wohngebäuden durch die Flut ist in urkundlicher Überlieferung nur selten zu finden: Das am Mühlstrom der Elster gelegene Jakobskloster in Leipzig musste 1343 seine Pfarreigebäude wiederherstellen (Posern-Klett 1868: 25), wie 1344 und 1345 auch der Bischof von Merseburg Witterungsschäden an seiner Kathedrale ausbessern ließ (Kehr 1899: 836, 853).

Insgesamt finden sich also viele Indizien für gegebenen Reparaturbedarf von (Wasser-)Infrastrukturen, der sich aber nicht in jedem Fall ursächlich auf die Hochwasserereignisse zurückführen lässt, auch wenn die zeitliche Koinzidenz vorhanden ist. Der aus dendrochronologischen Daten hergeleitete Rückgang der Bauaktivitäten in West- und Mitteleuropa, der aufgrund 


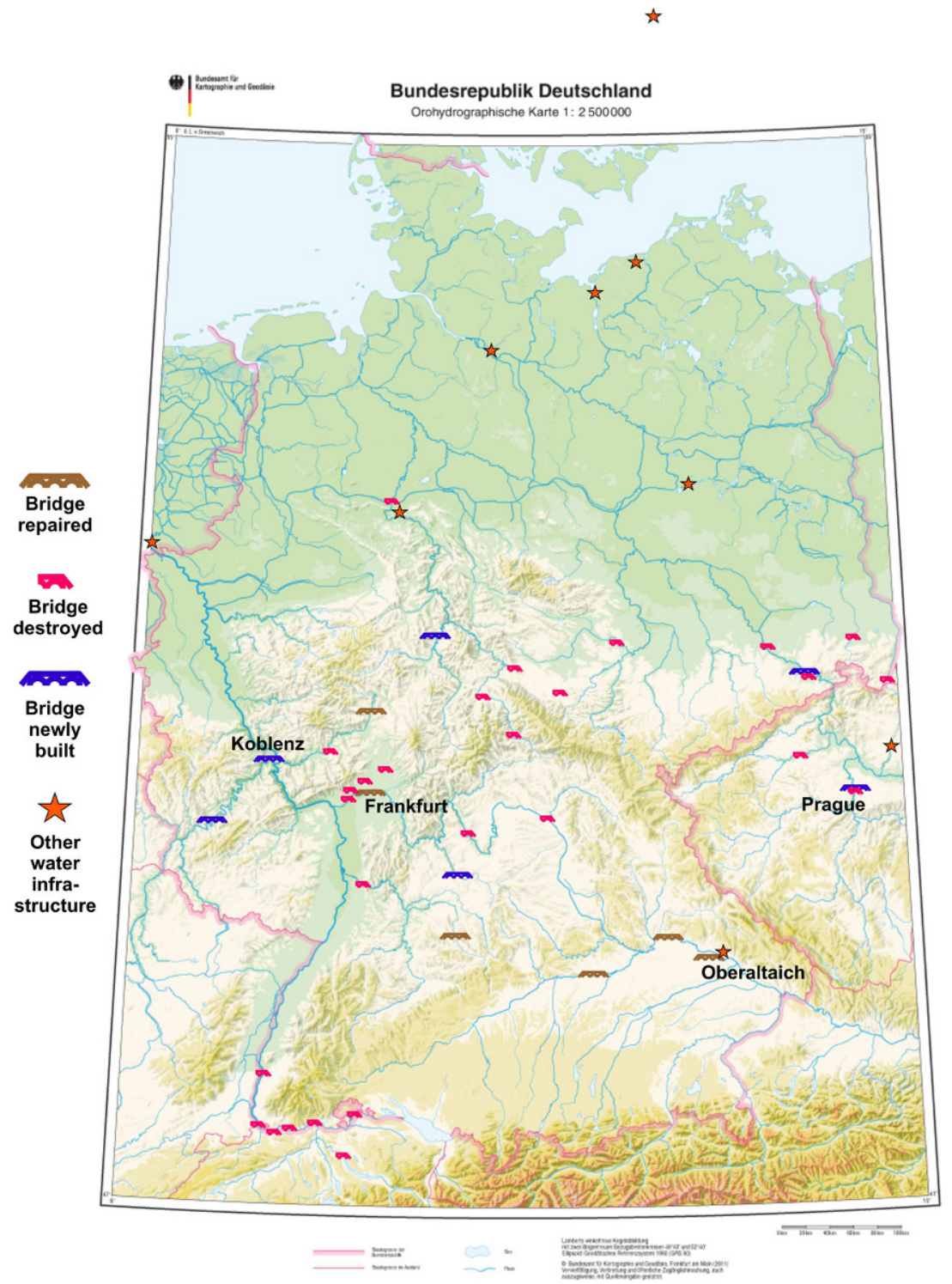

Abb. 4 Orohydrographische Karte Mitteleuropas im Maßstab 1:2.500.000 mit Angaben zu zerstörten, reparierten und neu gebauten Brücken sowie anderen Wasserinfrastrukturen der Jahre 1342/43, wie im Artikel diskutiert. (Karte: @ Bundesamt für Kartographie und Geodäsie, Frankfurt am Main/Adaption Martin Bauch)

der abnehmenden Baumfällungen ab circa 1340 begründet wurde (Charpentier Ljungqvist et al. 2018), lässt sich so noch nicht plausibel machen. Vielmehr gibt es einzelne Belege, die für eine verstärkte Nachfrage nach Bauholz sprechen, wie eine Urkunde zur Waldnutzung vom November 
1342, die die Entnahme von Feuerholz aus einem Wald erschwerte, während Eiche exklusiv nur für Baustellen genutzt werden durfte (Friese 1987: 214). Diese verstärkte oder zumindest unverminderte Nachfrage lässt sich mit dem Rückgang in den Fälldaten vielleicht vereinbaren, wenn man bedenkt, dass diese überwiegend aus in Wohngebäuden verbauten Hölzern (Charpentier Lungqvist et al. 2018: 83) resultieren und nur zum geringsten Teil aus hydraulischen Strukturen. Sollte also Bauholz einige Jahre nach den Flutereignissen der 1340er mit Vorzug für Brücken, Dämme und Mühlen genutzt worden sein, bei gleichzeitig stagnierender Bevölkerung aufgrund von Nahrungsmittelknappheit (siehe unten), wäre das Gesamtbild wieder stimmig.

\section{Erosion und Wüstungsprozesse}

Doch die Zerstörung von Wohn- und Wasserinfrastrukturen war nur ein Teil des Impacts der Hochwasserereignisse von 1342/43: Geomorphologen um Hans-Rudolf Bork haben seit langem auf ein herausragendes Erosionsereignis in der ersten Hälfte des 14. Jahrhunderts verwiesen, das sie in ganz Mitteleuropa nachweisen können und mit einem einzelnen Extremniederschlag in Verbindung bringen (Bork et al. 2011; Bork \& Bellstedt 2000; Bork 1988: 226-251). Die genaue zeitliche Verortung und die Identifizierung mit der Magdalenenflut 1342 erfolgte über den schriftlichen Befund (im Wesentlichen Weikinn 1958). Das Vorgehen und die Zuordnung auf genau ein Jahr ist nicht ohne Widerspruch geblieben (Seils 2000: 177-179; Bauer 1993). Allerdings deuten Warvenchronologien aus Eifelmaaren (Sirocko 2009: 173, Abb. 31.5) und dem Belau-See (Dreibrodth \& Wiethold 2015: 316-317) in dieselbe Richtung, wenngleich auch ihre Feindatierung nicht unabhängig von den Schriftquellen geschieht; dendrochronologische Lokalbefunde (Abb. 2a-f) zeigen hingegen, dass die Sommermonate 1342/43 fast überall zu den zehn feuchtesten des Jahrhunderts gehören. Im chronikalischen Befund stellt sich die Beschreibung von Erosion und späterer Ablagerung der Sedimente am Unterlauf der Flüsse als eine selten belegte Ausnahme dar (Leibniz 1711: 695; Schneider 1910: 225-226).

Dass die von Getreidemonokulturen geprägten Landschaften Mitteleuropas besonders anfällig für Erosion waren, ist keine Überraschung. Im urkundlichen Befund spiegelt sich dies nur gelegentlich wieder, etwa Anfang April 1342, als Zinszahlungen an den Bischof von Brandenburg nur dann ausbleiben durften, wenn die Bauern belegen konnten, dass die von ihnen bearbeiteten Felder von Flugsand bedeckt und daher wüst gefallen waren (Riedel 1869: I 13, 32). Man kann diesen Befund auch im Kontext der oben erwähnten, relativen Trockenperiode seit 1336 und vor allem des niederschlagsarmen Frühjahres 1342 sehen. Erosionsereignisse zeigen sich auch an unerwarteten Befunden in Urkunden, wie bei dem Streit zwi- 
schen dem Abt von Kainsheim und den Augustinereremiten in Esslingen vom 9. September 1342 um Boden, der vom Weinberg des Abtes auf das Gelände der Augustiner herabrieselte (Schreg 2013). Die zugrundeliegende Urkunde (Regest bei Diehl 1899: 368-369) beschreibt dies als eher regelmäßigen Vorgang: „Daz der Wingarte der an dem Berge ob dem vorgenan[nten] Convente gelegen ist taegelich uf sinen Convent rise und viele

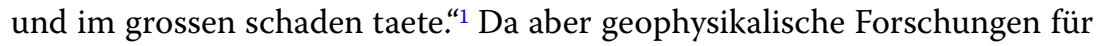
Südwestdeutschland auf großflächige Starkregenereignisse mit zugehöriger Erosion in diesem Zeitraum hindeuten (Beckenbach et al. 2013) und gerade an der nahe Esslingen gelegenen Schwäbischen Alb Hangrutschungen in Zeitlupe durchaus typisch sind (Bell 2007), kann hier plausibel von einer Verbindung zu den Starkregen 1342/43 ausgegangen werden. Nicht zuletzt hätte die vorausgegangene Trockenperiode im April/Mai 1342 das Eindringen von Wasser in die Erde durch Trockenrisse begünstigen können (ebd.: 107, 207). Baumringdaten machen klar, dass die ganze erste Hälfte der 1340er Jahre im Mittleren Neckarraum als ausgeprägt feucht gelten darf, was die Monate Juni-August angeht (Abb. 2b).

$\mathrm{Ob}$ die in diesem Beitrag untersuchten Ereignisse hinreichend massiv waren, dass sie zur Aufgabe von Siedlungen geführt haben, ist mit Gewissheit schwer festzustellen: Am Beispiel einer oberfränkischen Wüstung (Dotterweich et al. 2003) und auch im Fall der Dorfwüstung Winnefeld im Solling (Stephan 2011; Stephan 2013) ist der Zusammenhang von Extremniederschlägen des 14. Jahrhunderts und der Aufgabe von Siedlungen vorgebracht worden. Der zeitgleiche Schriftbefund zu Wüstungen ist hingegen wenig hilfreich, da nicht ersichtlich wird, seit wann die Siedlungen verlassen waren, obwohl es für die Jahre 1342-1345 zahlreiche Belege gibt (Schmidt 1878: 362; Boček 1860: 297; Hodenberg 1848: 69-70; Riedel 1869: I 3, 375; Schmidt 1885: 439; Emler 1892: 608; Riedel 1869: I 6, 467), im Fall der oberfränkischen Wüstung Friesen bei Kronach (Abb. 1) sogar für die unmittelbare Umgebung im Jahr 1343 (Schweitzer 1853: 118). Dass das Wüstfallen von Ortschaften Anfang der 1340er häufige Ereignisse waren, lässt sich vielleicht in aller Vorsicht aus dem mehrfachen Gebrauch einer identischen Formulierung schließen: „Aream sive locum ville [...] iam deserte damus" (Riedel 1869: I 18, 387).

Nähme man aufgrund der geomorphologischen Befunde also an, dass die gesellschaftlichen Konsequenzen durch die immense Erosion weitreichend gewesen sein müssten, so wird das schon im Hinblick auf Wüstungen uneindeutige Bild noch schwieriger, wenn wir eine zweite denkbare Konsequenz des weggespülten Ackerbodens suchen: Teuerung und Hunger in den Folgejahren aufgrund der zerstörten Ernte 1342 sowie verringerter Anbauflächen. Jüngere Forschungen zu vormodernen Hungerereignissen (Überblick bei Collet \& Schuh 2017) machen dabei deutlich, dass diese 
immer als Verflechtung natürlicher und anthropogener Faktoren zu verstehen sind.

\section{Teuerung und Hunger}

Die Beschreibungen der Magdalenenflut in den narrativen Quellen erwähnen vielfach die Zerstörung der Ernte und den Schaden für die Feldfrüchte durch die Extremniederschläge und das Hochwasser (Weikinn 1958: 200, 203, 206, 212-214). Dies gibt wichtige Hinweise, dass die Getreideernte zum Zeitpunkt des Einsetzens der Starkniederschläge noch nicht flächendeckend eingebracht worden war. Der Ablauf des Landwirtschaftsjahres (Pfister 2005) ist entscheidend dafür, ob die natürlichen Extremereignisse im Juli 1342 die Nahrungssicherheit im folgenden Jahr beeinflussen konnten.

Doch wenn wir zeitgenössische Chroniken suchen, die tatsächlich Teuerung und Hunger in den Folgejahren unabhängig vom eigentlichen Hochwasserbericht erwähnen, bleibt etwa nur eine nordwestdeutsche Quelle, die reduzierte Nahrungsverfügbarkeit bereits 1342 (Ficker 1851: 48) erwähnt, während für Süddeutschland erst für 1343 von einer massiven Hungersnot gesprochen wird. Johann von Winterthur berichtet von Hungertoten im Südwesten des Reichs und in Niederbayern, die er kausal mit dem Starkregen des Vorjahres verbindet (Baethgen 1924: 200, 205). Im Salzburger Land (Wattenbach 1851: 829) und in Niederösterreich (Wattenbach 1851: 673) wird ebenfalls von enormer Teuerung des Getreides berichtet. Aus narrativen Quellen drängt sich daher der Eindruck auf, nur Süddeutschland, ja eigentlich nur die Gebiete südlich der Donau hätten 1343 mit einer relevanten, aber eben regional begrenzten Hungersnot zu kämpfen gehabt.

Wenn wir allerdings Indikatoren für Nahrungsmangel in einer erweiterten Quellenbasis suchen, also in Urkunden und administrativen Aufzeichnungen, zeigt sich ein anderes Bild: Ein Abgabenverzeichnis des Chorherrenstiftes St. Gumbertus in Ansbach bietet insgesamt nur wenig Angaben zu Korn, aber der Stiftsbesitz in Windsheim lieferte 60 Prozent weniger Getreide als erwartet: „Wenigen Winsheim der hof. Ist geben ume vierzig malter. Item des ist worden zehendalp malter roken, sehs malter dinkels an drey metzen" (Schultheiss 1963: 85). Der Nachsatz ist von selber Hand, aber mit hellerer Tinte nachgetragen, wie am Original zu erkennen ist was für eine Ergänzung nach der spärlichen Ernte 1342 spricht. Im selben Jahr fallen nordöstlich der Elbe eine Reihe von Regulierungsmaßnahmen im Ernährungsbereich auf: Die Stadt Garz an der Oder verbot Getreidespekulation und hielt ihre Bürger an, sich einen Kornvorrat für ein Jahr anzulegen. Mehl und Getreide durften nicht mehr ausgeführt werden (Conrad 1990: 186-187). Im September 1342 sicherte sich die Bäckergilde von Treuenbrietzen städtische Entschädigung, wenn ihre Mitglieder wegen 
des Mangels an Korn oder defekter Getreidemühlen kein Brot mehr herstellen konnten (Riedel 1869: I 9, 369). Die Metzger von Wismar wurden Ende August 1342 von ihrem Rat dahingehend reguliert, dass sie importiertes Rindfleisch nur zu festgesetzten Preisen verkaufen durften (Verein für mecklenburgische Geschichte und Althertumskunde 1875: 401-402). Und auch am Niederrhein sah die Lage nicht besser aus: Herzog Rainald II. von Geldern konnte am 6. Februar 1343 die Leibrenten für seine Schwestern Isabella und Philippa, Klarissen zu Köln, nicht bezahlen, weil „dit jaer“ gekennzeichnet war durch „misvasse ende verderfenisse der vrucht, die comen syn van groter watervloet" (Sauerland 1905: 62-63). Den Zahlungsversprechungen des Herzogs für die Zukunft trauten die Schwestern, eine davon Äbtissin des Konvents, dabei nicht, sondern sie versuchten über den Papst in Avignon ihre Besitzansprüche gegenüber ihrem Bruder einzuklagen, wobei ihnen im Juli von der Kurie immerhin ein Zugriff auf die Nachlässe aller Konventsmitglieder bestätigt wurde (ebd., Nrr. 163, 198). Im September 1343 gewährte der Papst sogar einen Ablass zugunsten des Weiterbaus der Kölner Klarissenkirche, initiiert von der Äbtissin Isabella von Geldern und ihrer Schwester Philippa, da sie dieses Jahr Verluste an ihrem Einkommen von $4000 \mathrm{fl}$. „propter subitam inundationem aquarum“ erlitten hätten (ebd.: 103).

Die zeitliche Verdichtung dieser Befunde deutet darauf hin, dass Nahrungsmittel an vielen Orten Mitteleuropas knapp waren durch Misswuchs und hochwasserbedingte Zerstörung der Feldfrüchte zugleich. Und die Belege lassen sich weiter ergänzen: Die Kanoniker von St. Thomas in Leipzig stellten zwei ihrer Stiftsherren im August $1342 \mathrm{ab}$, immer für die Füllung der Vorratskeller mit Korn, Brot und Bier zu sorgen (Posern-Klett 1868: 82). Dass dahinter mehr als die Neuvergabe einer Standardaufgabe liegen könnte, zeigt die erstaunliche Regelung des Domkapitels von Meißen vom 23. Oktober 1342, wenn es um die künftigen Termine der Auszahlung von Getreide- und Geldzehnten ging. Diese wurden von der ungewöhnlichen Beifügung ergänzt, dass „wer auch immer lebend den Jakobstag“ erreiche, also den 25. Juli, erhalte das Korn ausgehändigt, und wer den Michaelstag (25.09.) oder auch Walpurgis (01.05.) erlebe, erhalte das Geld ausbezahlt. Diese Formulierung vivendo attingeret ist einmalig in der Überlieferung des Domkapitels und auch kein biblisches oder sonstwie herleitbares Zitat. Sie kann daher wohl als Indiz einer tiefgehenden Verunsicherung angesehen werden; ein Bedrohungsgefühl, das durchaus mit dem Erlebnis der Juliflut zusammenhängen könnte, die vor Ort und im nahen Dresden die Elbbrücke vernichtet hatte. Und im Jahr 1343 blieb die Situation schwierig, wie wir Quellen aus dem Zeitraum September bis Dezember entnehmen können - Monate, die in einem normalen Jahr aufgrund der neuen Ernte eine entspannte Ernährungslage bis weit ins Frühjahr des nächsten Jahres 
einläuteten. Doch am 20. September 1343 waren die Zisterzienserinnen von Ichtershausen in Thüringen gezwungen, um eine mehr als das pure Leben erhaltende Ernährung aus Wasser, Brot und Gemüse zu betteln (Overmann 1929: 96). Nicht besser ging es Schwestern desselben Ordens aus Börstel bei Osnabrück, denen im September und erneut im Dezember 1343 das Geld zum Kauf von Nahrungsmitteln fehlte und erst eine zweimalige Spende ihrer Äbtissin schuf Abhilfe (Rölker \& Delbanco 1997: 112-113, 118-119). Den Eindruck großflächiger Nahrungsmittelknappheit im Reich bestätigt ein Exportverbot für Getreide in Richtung Kontinent, das Ende Dezember 1343 König Eduard III. von England erließ - mit der Ausnahme der deutschen Lande, die Mangel an Korn litten, „ubi viget caristia bladorum ut dicitur" (Höhlbaum 1886: 11).

Neben dem unzweifelhaften Impact der Hochwasserereignisse auf Infrastrukturen konnte der geowissenschaftlich erhobene Befund der Erosion durch Schriftquellen zumindest ergänzt werden, und die Erweiterung des Quellenspektrums im Hinblick auf Hunger und Teuerung gibt ein relativ dichtes Bild über bedrohliche Nahrungsmittelknappheit in den Jahren 1342/43 nicht nur im äußersten Süden des Reichs, sondern überall zwischen Niederrhein und Oder.

\section{Adaption von Wasserinfrastrukturen und Regulierungen der Nahrungsmittelversorgung sowie soziale Konflikte}

Doch inwiefern folgte diesem skizzierten Schadensbild nicht nur ein Wiederaufbau bzw. eine Linderung des Mangels, sondern eine tatsächliche Adaption in Richtung einer Prävention aufgrund der gemachten Flut- und Mangelerfahrung? Und wie konnte dieses bereits weiter oben diskutierte Umweltwissen zweiter Ordnung aussehen?

\section{Anpassung der Infrastrukturen}

Das prominenteste Opfer der 1342er Hochwasser war die Prager Judithbrücke, deren Wiederaufbau erst 1348 beschlossen und ab 1357 realisiert wurde (Šefců 2007). Der heute als Karlsbrücke bekannte Neubau zeichnet sich dadurch aus, dass die Köpfe der Brückenpfeiler gegen die Fließrichtung der Moldau verstärkt worden waren, mutmaßlich um Eisgang und sonstigem Treibgut besser zu widerstehen. Dass aber für die spätere Karlsbrücke nicht ohne weiteres die 1340 vollendete Steinbrücke von Roudnice nad Labem als Vorbild herangezogen werden kann, ist vor allem darin begründet, dass der erstmals 1351 erwähnte Brückenbaumeister Otto mit seiner Expertise, hätte er sie vor 1340 in Roudnice gewonnen, wohl kaum über 
zehn Jahre beschäftigungslos in Böhmen verblieben wäre (Bauch 2017: 996). Denn der Technologietransfer erfolgte bereits im Jahr 1333 durch einen französischen Brückenbaumeister Guillaume, der böhmische Handwerker ausbildete, so dass sie bis 1340 den Bau vollenden konnten (Cihla \& Panáček 2011). Starke Sommerniederschläge muss es laut dendrochronologischem Befund in Böhmen seit 1339 gegeben haben (Abb. 2d).

Doch innerhalb der luxemburgisch regierten Territorien gab es einen früheren Steinbrückenbau, der in der Forschung relativ wenig Beachtung gefunden hat: die im Juli 1343 erstmals erwähnte Balduinbrücke in Koblenz, die von Erzbischof Balduin von Trier durch die Erlangung mindestens eines päpstlichen und die Gewährung eines erzbischöflichen Ablasses finanziert werden sollte. Diese Ablässe zielten auf Spenden aller Art für die Bauhütte $\mathrm{ab}$ und generierten diese in den nächsten Jahren auch reichlich (Lorenz-Corswarem 1949: 16-18; Sauerland 1905: 82; Winkelmann 1885: 814). Der Bau hatte 1343 schon eingesetzt, und als Begründung für die Errichtung der Brücke wurden die verschiedenen Hochwasser genannt, die die Pilger auf ihrem Weg nach Aachen und Rom gefährdet hätten (Mindermann 2004: 505); was die dendrochronologische Niederschlagsrekonstruktion zumindest für den Sommer 1342 bestätigt (Abb. 2c). Als Brückenbauherr war Erzbischof Balduin wohl zeitgleich in Trier tätig (Standfuss 1988: 404), wo er eine Holzbrücke durch eine Steinbrücke ersetzen ließ, während die Existenz eines Vorgängerbaus in Koblenz (Sauer 1988: 408) unwahrscheinlich ist, weil Balduin selbst betont, dass der Fluss nur zu Schiff überquert werden konnte: „flumen Moselle, quod est magnum et navigabile ac sine navis subsidio communiter transiri non potest" (Sauerland 1905: 82). Der genaue Baubeginn der Balduinbrücke liegt also im Dunkeln, aber es gibt belastbare Indizien, dass Hochwasserereignisse Bau und Gestaltung der Brücke beeinflussten. Der zweite Pfeiler der Brücke von Süden - der Koblenzer Seite - her, wurde von Unterwasserarchäologen nach seiner Zerstörung im Zweiten Weltkrieg untersucht und zeigt Anomalien. Statt der bei den restlichen Pfeilern üblichen 170-230 Gründungspfähle (Abb. 5a) weist er nur 66 auf, die zudem vielfach um 45 Grad verdreht sind (Abb. 5b). Ebenso wurden abgesunkenes Mauerwerk und eine stabilisierende Holzverschalung um den Pfeiler herum gefunden - alles Hinweise auf ein starkes Hochwasser oder Eisgang, die auf den im Bau befindlichen Pfeiler einwirkten (Schneider 1949: 7-8). Unter der Voraussetzung, dass der zweitsüdlichste Pfeiler auch einer der ersten im Bau befindlichen war - immerhin konnte stadtseitig einfacher Material und Handwerker für den Bau herangeschafft werden -, und unter Berücksichtigung, dass die Errichtung von Pfeilern in Flachgründung nur während Niedrigwasserperioden im Sommer vorangetrieben werden konnte, spricht der Befund also für eine Beschädigung des entstehenden Pfeilers im Sommer 1342 oder 


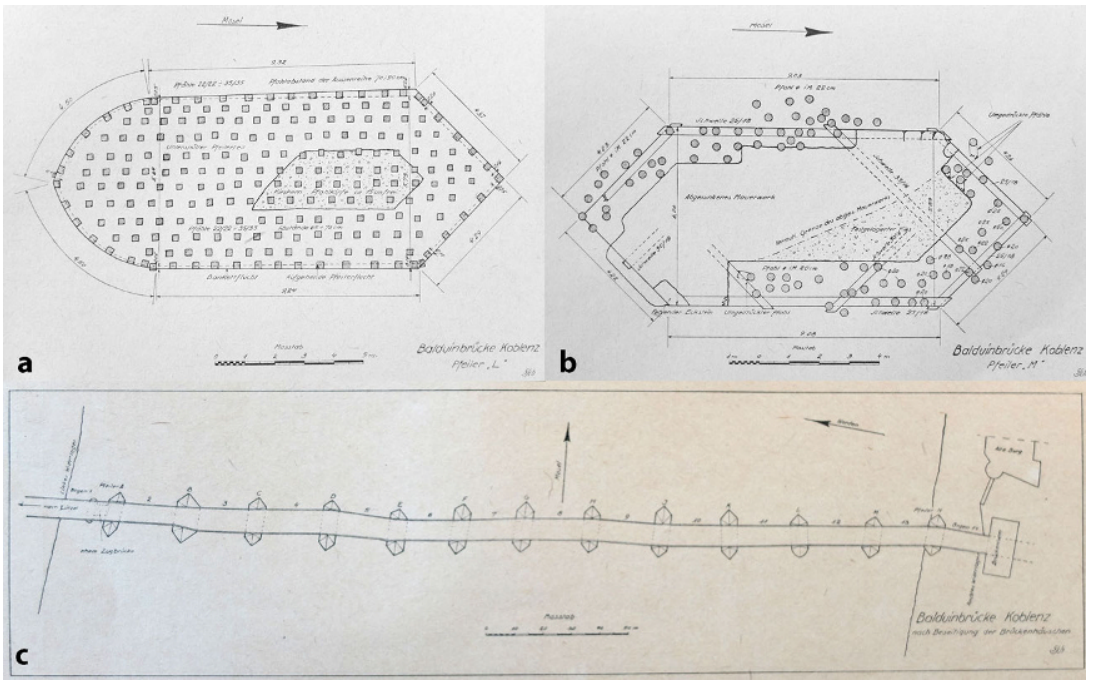

Abb. 5 a Archäologischer Befund beim dritten Pfeiler der Balduinbrücke (L) von Koblenz her, angenommener Normalfall der Pfahlgründung. b Skizze des Befundes des zweitsüdlichsten Brückenpfeilers $(\mathrm{M})$ bei seiner archäologischen Untersuchung während des Wiederaufbaus 1946-49. c Draufsicht der Balduinbrücke, Koblenzer Seite rechts. Die von der Achse der Brücke abweichende Ausrichtung der Pfeiler ist deutlich zu erkennen, am deutlichsten bei dem oben diskutierten, mutmaßlich von Hochwasser beschädigten Pfeiler M (2. v.r.). (Quelle: Schneider 1949, Abb. 6-7)

1343. Auch wenn keine regionalen Schriftquellen von einem Moselhochwasser oder Starkniederschlägen in diesen Jahren berichten, zeugen doch die Flutlagen in den Sedimentwarven des nördlich des Flusses liegenden Schalkenmehrener Maars (Sirocko 2009: 173, Abb. 31.5) von ausgeprägten Niederschlägen in den 1340er Jahren, mit einer mächtigen Varve, die dem Jahr 1342 zugeordnet wird. Plausibel ist dies durch eine nur wenige Jahre jüngere Lage mit Insektenresten, die mit hoher Wahrscheinlichkeit dem Heuschreckeneinfall in Mitteleuropa 1338-39 zugeordnet werden können (Bauch 2017: 992-993; Sirocko 2009: 167). Bestätigung finden diese Indizien in der dendrochronologischen Rekonstruktion von Niederschlägen für die Region (Abb. 2c), die 1342 als sechstfeuchtesten Sommer des Jahrhunderts anführen. Und noch ein weiterer Hinweis bestärkt den Eindruck, dass auf die im Bau befindliche Balduinbrücke ein extremes Hochwasserereignis eingewirkt haben könnte: Die 13 Pfeiler stehen nicht rechtwinkling zur Achse der Brücke, sondern jeder für sich leicht versetzt (Abb. 5c): „Es kann behauptet werden, dass die Stellung sämtlicher Pfeiler der Brücke von genauer Strömungsbeobachtung der Brückenbaumeister Zeugnis ablegt" (Schneider 1949: 3). 
Wenn also bei der Balduinbrücke fast von Baubeginn an eine Wiederkehr von extremen Hochwassern bedacht wurde, und der kurfürstliche Bauherr zu Koblenz engste verwandtschaftliche Beziehungen $\mathrm{zu}$ seinem Neffen in Prag pflegte, so scheint ein Expertenaustausch von der Mosel an die Moldau nicht unwahrscheinlich, wie wir ihn etwa im diplomatischen Bereich mit dem kurtrierischen Sekretär Rudolf Losse, seit 1347 Rat von Karl IV., bis in die höchsten Ebenen der karolinischen Kanzlei vorfinden (Sauerland 1905, Nr. 215-216). Und auch die Balduinbrücke selbst war im Blickfeld des Kaisers, der die wohl spätestens ab 1359 begehbare, da mit einem Zoll belegte Brücke (Schneider 1949: 13), für Ausbesserungen und in Richtung ihrer Vollendung hin unterstützte (Hohensee et al. 2013, Nr. 467). Zwar war die bauliche Gestaltung der Karlsbrücke auch in Hinblick auf Hochwasserschutz eine andere als die Lösung, die für den Koblenzer Fall getroffen wurde. Aber die Balduinbrücke ist doch das prominenteste Infrastrukturprojekt in luxemburgischem Auftrag vor dem Jahr 1357, und sie stellt wohl eine direkte Adaption einer bereits vorab geplanten Brücke auf die Hochwasserereignisse der Jahre 1342/43 dar. Der Primärzweck der Brücken in Koblenz und auch in Prag lag allerdings nicht im Hochwasserschutz, dementsprechend kann man sie auch nur bedingt als Ergebnisse herrscherlicher Krisenprävention betrachten.

Anders ist die Lage beim ersten bekannten Donaudurchstich in der Nähe von Straubing. Dort war das Benediktinerkloster Oberalteich in unmittelbarer Nähe einer Stromwindung gelegen, die mutmaßlich $1342 \mathrm{zu}$ einer Überflutung der Klosteranlage durch die Donau geführt hatte. In jedem Fall zeigen die lokalisierten Baumringrekonstruktionen (Abb. 2f), dass 1342-1345 prononciert feuchte Sommer vorherrschten. Ein künftige Entlastung versprechender Durchstich zwischen den Ortschaften Reibersdorf und Bogen auf über zwei Kilometern Länge ist mit Fug und Recht als direkte Reaktion auf die Hochwassererfahrung verstanden worden, die zudem kaiserliche Unterstützung erfuhr (Rohr 2007: 364-365). Das Mitte des 14. Jahrhunderts geschaffene Bett der Donau ist noch heute in Nutzung. Aus frühneuzeitlicher Historiographie des Klosters wurden die konkreten Infrastrukturarbeiten rekonstruiert (Neueder 2012: 47-49), die sich aber fast alle aus urkundlicher Überlieferung bestätigen lassen. Darüber hinaus beschreibt die zeitgenössisch in Oberalteich entstandene Chronik der Herzöge von Bayern detailliert die immens teure, das Kloster mit 10.000 Pfund Regensburger Pfennigen belastende, über zehn Jahre in Anspruch nehmende Umleitung des Stromes in ein neues Bett (Leidinger 1918: 169-170). Hatte Abt Wolfgang (1338-46) vor 1341 noch die Verschuldung des Klosters reduzieren können (Neueder 2012: 48), änderte sich mit Beginn der Bauarbeiten vieles: Schon ab 1343 erhielt das Kloster vom Domkapitel Augsburg Grundbesitz bei Niederöbling zur Unterstützung der Aushubar- 
beiten (Monumenta Boica 1775: 176-179). Eine kaiserliche Urkunde von 1344, die dem Kloster weitere Bauernhöfe zueignete, nannte das allgemeine Wohl und den imperialen Willen als Begründung der Infrastrukturmaßnahme: „Damit sie die Tunau abgrabent, den sie von unserem haiss, und gebot, und durch gemainen Nuz, und frumm [...] pauent, und machent" (ebd.: 181-182). Weitere Vogteirechte über Örtlichkeiten gingen an das Kloster, darunter über den Ort „Anschütt“ nahe des Bogenbergs über der Donau (ebd.: 182-183) - eine Bezeichnung, die auf ein Alluvium, also angeschwemmte Flusssedimente, hindeutet. Dass der Durchstich tatsächlich ein Projekt von Kaiser und Kloster war, zeigen weitere Schenkungen Ludwigs an Zinsrechten und Bargeld in den Jahren 1346/47 (ebd.: 186-188). Auch nach Vollendung des Durchstichs zahlte das Kloster bis 1359 „von des grabens wegen“ (ebd.: 195-197) Schulden zurück. Noch in den 1360er Jahren schwelten Rechtsstreitigkeiten mit Bauern von Reibersdorf über Weiderechte auf einer ehemaligen Donauinsel beim Weiler Lenach. Der Abt inspizierte die trocken gefallene Insel mit Fischern und Bauern und man fand zum Kompromiss, dass die Bauern ihr Vieh auf dem neuen Anschütt, dem verlandeten Donauarm östlich Lenach weiden durften, während Wiesen südlich des Altarms beim Kloster verblieben (ebd.: 201-204). Der Oberalteicher Donaudurchstich ist nicht nur die erste bekannte Flussbegradigung der Donau; er zeigt auch den Kaiser Ludwig IV. als bayerischen Landesherrn in einem längerfristigen Engagement als Initiator einer komplexen Wasserinfrastruktur, die keinen ökonomischen Hintergrund hatte, sondern nur der Erhöhung der Hochwassersicherheit für Oberalteich dienen konnte. In dieser Funktion als tatkräftiger, finanziell engagierter Beförderer der öffentlichen Sicherheit vor Naturkatastrophen ist Ludwig der Bayer ein Pionier unter den nordalpinen Herrschern im Spätmittelalter. Die intendierte Vermeidung von Opfern und Zerstörungen war eigentlich erst seit dem ausgehenden 15. Jahrhundert eine Aufgabe der Regierenden, zumindest nördlich der Alpen (Labbé \& Schenk 2018).

Typischer, weil rein passiv in seinem Vorgehen ist Karl IV. im Jahr 1343, noch als Markgraf von Mähren Regent im böhmischen Königreich. In einem undatierten Dokument (Haas 1954: 76; Tadra 1880: 510-512) aus der Kanzlei des Ernst von Pardubitz, das aber aufgrund der Titulatur Karls als capitaneus regni Bohemie, nur gebräuchlich bis Mai 1343 (Boček 1860: 346), in die Zeit unmittelbar nach der Magdalenenflut fallen muss, wird dem nordböhmischen Adligen Jan von Dražic die Verlegung seiner Marktsiedlung Benatek an der Iser erlaubt. Sie sei in verschiedenen, nicht genau ausgeführten Formen verheert worden - dass dazu ein Hochwasserschaden gehören könnte, impliziert die neue Lage der Siedlung auf dem Hügel Hradiště oder Hrobye am rechten Ufer der Iser, während die alte Siedlung Staré Benatky am linken Ufer sehr tief lag (Bahlke et al. 1998: 27). Dass 
Böhmen Anfang der 1340er Jahre prononciert feuchte Sommer aufwies, zeigt der Baumringbefund (Abb. 2d). Die Siedlungsverlegung als Reaktion auf ein natürliches Extremereignis ist hier also nicht gesichert; in jedem Fall ist es aber nicht der Landesherr, der aktiv wird; er erteilt nur seine Genehmigung.

Dass es nicht immer der Kaiser sein musste, der aktiv Wasserinfrastrukturen förderte, um künftig die Sicherheit zu erhöhen, zeigt ein letztes, instruktives Beispiel vom Niederrhein. Im bereits eingangs hinsichtlich der zeitlichen Ausdehnung der Flutereignisse erwähnten Herzogtum Geldern wurden die Ritter und Knappen der Betuwe von Herzog Rainald II. im Juni 1343 - also vor Durchgang des Sommerhochwassers im Juli zusammengerufen, um in Nijmwegen über kollektiven Deichbau zu beraten (Janssen 1970: 234). Dabei gibt es Indizien für solch organisierten Hochwasserschutz am Niederrhein seit dem 13. Jahrhundert (Schreiner 1995: 44) und bereits in den 1320er Jahren waren in Geldern verschiedene Deichschauen - also für den Deichbau und dessen Sicherung verantwortliche Gemeinschaften - eingerichtet worden, einige davon unter aktiver Beteiligung desselben Herzogs (ebd.: 46-48; Schreiner 2001). Wie es aussehen konnte, wenn eine solche Intervention unterblieb, zeigt ein Streit zwischen den Elbanliegern Neuengamme und Kirchwerder, wer für die Unterhaltung des Flussdeiches zuständig war (Mindermann 2004: 532). Zeitlich knapp vor der Deichschau von Geldern liegt aber die bemerkenswerte Initiative des Dietrich von Hoorn, Herr von Kranenburg und Neffe des Grafen von Kleve, einem unmittelbaren Nachbarn des geldrischen Herzogs. Am 2. Februar 1343 erließ er das erste in vollständiger Fassung überlieferte Deichrecht (Teschenmacher 1721: 17-19) für seine kleine Herrschaft, eingeklemmt zwischen Kleve im Westen, Geldern im Osten, dem Rhein im Norden und der Maas im Süden. Das Interesse des kleinen Territorialherren an Sicherung seines Gebiets gegen Hochwasser ist also offenkundig. Auch hier wie im benachbarten Geldern waren schon vor 1343 Ansätze einer Deichbauorganisation gegeben, und die sogenannte hanntveste von Cranneborch umfasste nicht nur eine Reorganisation von Infrastruktur nach holländischem Vorbild, sondern auch eine geographische Erweiterung des ihr unterliegenden Gebiets (Schreiner 1995: 117-118). Bemerkenswert ist dabei die Regelung, dass der Landesherr für Deiche und Entwässerungswerke finanziell einspringen musste, für die kein pflichtiger Grundeigentümer mehr gefunden werden konnte (ebd.: 121). In diesem Akt überschneidet sich eine konkrete infrastrukturbildende Reaktion von landesherrlicher Seite auf das Flutereignis mit einer abstrakt-zukunftsorientierten, normativen Adaption, die freilich beide präventiven Charakter hatten. 
Normative Reaktionen: Nahrungsmittelregulierung, Brandschutz und "Schlechtwetterklauseln"

Bei der Skizzierung des Impacts der Flutereignisse 1342/43 auf die Verfügbarkeit von Nahrung - meist Getreide - weiter oben, ließen sich bereits eine Reihe von meist kommunalen Regulierungsmaßnahmen anführen, die auf allgemeine Lebensmittelknappheit hindeuten. Doch die elaborierteste Regelung von Nahrungsangelegenheiten finden wir in den sogenannten „Polizeisätzen“ von Würzburg (Hoffmann 1955), einer Stadt, die nachweislich hart von der Hochwasserwelle im Juli 1342 getroffen worden war (Borchard 1988; Böhmer 1843: 469). Bischof Otto II. von Wolfskeel erließ diese statutenähnlichen Vorschriften für seine Stadt, und Michael de Leone, seit 1336 bischöflicher Protonotar und damit vermutlich eng vertraut mit deren Erstellung, nahm sie in sein bekanntes Hausbuch auf, das bis 1355 entstand (Brunner 1983; Kornrumpf \& Völker 1968: 66-107). Die ältesten dieser städtischen Ordnungen für Würzburg sind in drei Teilen überliefert: Einem ersten ohne Datum, einem zweiten vom 9. Juli 1342, einem dritten vom 27. April 1343. Dabei sind die geregelten Inhalte aufschlussreich: Der zweite Teil der Polizeisätze, noch vor der Flutwelle im Juli 1342 erlassen, reguliert den Handel mit Holzkohle, Ziegeln, importiertem Wein, Schwertern, Messern, und das Geschäft der Metzger, Gastwirte und Müller, aber auch das Wiegen von Mehl und die dafür genutzten Maße (Hoffmann 1955: Nr. 72-88). Dies alles sind noch keine spezifischen Indikatoren von Teuerung und Mangel bei Lebensmitteln. Anders ist die Lage im April 1343, als der dritte Teil der Polizeisätze erlassen wird, der die Arbeit der Bäcker bis ins feinste Detail regelt: Sowohl Qualität der Waren wie deren Preis wird reguliert, Kontrollmöglichkeiten für die Verbraucher werden aufgezeigt und unehrliche Bäcker sehen sich mit massiven Sanktionen konfrontiert (ebd.: Nr. 89-95). Höchstpreise für Mehl aus Weizen und Hafer, aber auch für Salz werden definiert (ebd.: Nr. 99-102). Nicht zuletzt ist es Winzern verboten, Wein aus anderen Früchten denn Trauben herzustellen (ebd.: Nr. 98); ein möglicher Indikator auf eine schlechte Weinernte im Herbst 1342. Der erste, nicht explizit datierte Teil der Statuten zielt vor allem darauf ab, alle Arten von Spekulation mit Grunderzeugnissen zu unterbinden: Heimischer wie importierter Wein, Brot, Bauholz, Holzkohle, aber auch Käse, Eier und Wild, getrockneter wie frischer Fisch, Krebse, Obst und sogar Tierfutter wie Heu, Gras und Hafer werden hier genannt (ebd.: Nr. 13-14, 20-31). Dabei ist völlig offen, ob der erste Teil wirklich früher entstanden ist als die beiden anderen; die Forschung positioniert sich dazu nicht (Ruland 1851: 67-73); doch am Manuskript des Hausbuches haben zwischen 1343 und 1350 sieben verschiedene Schreiber gearbeitet, so dass die Reihenfolge im Hausbuch nicht zwingend die chronologisch 
richtige Anordnung der Polizeisätze reflektiert, gerade wenn Datierungen fehlen.

Ein kausaler Zusammenhang zwischen der Entstehung der Würzburger Polizeisätze und den Ereignissen der Jahre 1342/43 ist in aller Eindeutigkeit jedoch nicht herzustellen. Aber der in diesem Beitrag vielfach ausgeführte zeitliche Kontext macht - neben einem unabhängig von den Extremereignissen immer vorhandenen Regulierungsbedarf wirtschaftlicher Aktivitäten in spätmittelalterlichen Städten - einen Einfluss der Hochwasserereignisse auf die bischöfliche Gesetzgebung zumindest plausibel. Die lokale Niederschlagsrekonstruktion aus Baumringen (Abb. 2e) zeigt jedenfalls eine Tendenz zu feuchten Sommern in der ersten Hälfte der 1340er Jahre, ohne dass jedoch die Jahre 1342/43 besonders herausragten.

Ähnlich gelagert ist, im Hinblick auf Korrelation und Kausalität, ein Erlass Kaiser Ludwigs des Bayern, der am 8. Mai 1342 zusammen mit dem Rat der Stadt München beschloss, dass alle Neubauten seiner Stadt München in Stein auszuführen seien, soweit die Bauherren dies finanziell leisten konnten. In jedem Fall war die Stroh- und Holzschindeldeckung von Dächern untersagt - selbst bei Scheunen und Gartenhäusern -, nur noch gebrannte Ziegel waren zulässig (Menzel 2003: 241-242). Entscheidend ist hier wiederum die zeitliche Koinzidenz: Zwar war die kaiserliche Residenz München schon 1327 durch Feuer zerstört worden, doch der verheerende Stadtbrand von Landshut hatte nur zwei Tage zuvor stattgefunden. Dies kann in München nicht ohne Beachtung geblieben sein; ob man von den drei weiteren Stadtbränden im Reich in den Vormonaten wusste, ist nicht zu beantworten.

Befriedigende Antworten auf die Frage, ob Regulierungen normative Reaktionen auf Extremereignisse waren oder eher kontinuierliche Bedürfnisse sich zunehmend ausdifferenzierender Gesellschaften befriedigten, würde nur eine verlässliche Chronologie solch regulierender Eingriffe über längere Zeiträume geben. Bis diese vorliegt ist schwer einzuschätzen, was es bedeutete, wenn norddeutsche Kirchenfürsten versuchten, ihre offenbar bedrohten Einkünfte zu sichern - so erließ Bischof Daniel von Verden im März 1344 Synodalstatuten, die gegen die Reduktion oder völlige Vermeidung von Zehntabgaben auf Korn und Rinder gerichtet war (Mindermann 2004: 528-529). Gleiche Mahnungen sprach der Erzbischof von Bremen im selben Monat aus (Ehmck \& von Bippen 1876: 503-504). Die selbstverständliche Pflicht, dass Zehnten auch bezahlt werden mussten, war offenbar so unsicher geworden, dass sie noch auf lokaler Ebene, etwa vom Kloster Osterholz in Niedersachsen (Jarck 1982: 117-118), eindringlich wiederholt wurden.

Allerdings lassen sich auch Kniffe in der rechtlichen Regelung von landwirtschaftlichen Abgaben finden, die man als „Schlechtwetterklauseln“ be- 
zeichnen könnte: „Sol auch das kuentbare sin ob von hagel ungewitter misseweges" (Veesenmeyer \& Bazing 1898: 235) - die Schenkung lokaler Adliger an das Ulmer Hospiz der Deutschherren im August 1342 war nur solange verpflichtend, bis Hagel, Unwetter oder „Misswuchs“ die Ausgangsbedingungen änderten. Solcherlei Ausschlussbedingungen finden sich häufig in Verträgen der Jahre 1343-45: Die Äbtissin des Frauenstifts Essen hatte sich 1343 innerhalb von zwei Wochen persönlich von möglichen Unwetterschäden zu überzeugen (Schilp 2010: 304). Wegen Schlechtwetters ausbleibender Weinzehnt in Wetzlar konnte im nächsten Jahr nachgeliefert werden (Sponheimer 1943: 227). Bei Uelzen sah man 1344 ein Wüstfallen durch Brand, Raub oder andere Faktoren vorausschauend als Grund für ein Erlöschen des Getreidezehnten (Vogtherr 1988: 54). Und als ein Bürger von Landshut 1345 einen Bauernhof außerhalb der Stadtmauern pachtete, wurde eine Anpassung seiner Zahlungen an mögliche Flutschäden ebenso definiert wie eine Pflicht zum Wiederaufbau des Hofes im Fall totaler Zerstörung durch Hochwasser (Herzog 1963: 276); dies kann auch nicht verwundern, zeigen doch die Dendrodaten, dass die Sommer der ersten Hälfe der 1340er Jahre fast durchweg äußerst feucht waren (Abb. 2f). Ein Risikobewusstsein für natürliche Extremereignisse war also deutlich vorhanden in Rechtstexten, die nach 1342/43 verfasst wurden - nur ist bisher nicht zu beantworten, um wie viel stärker es war als noch wenige Jahre zuvor.

\section{Fazit: (Erst-)Zumessung von Kritikalität für Infrastrukturen und Daseinsvorsorge nach dem compound event der Jahre 1342/43}

Betrachten wir nach dieser Tour d'horizon abschließend, ob die drei selbstgestellten Ziele des Beitrags als erfüllt betracht werden können.

Zum ersten ist unser Bild über den Verlauf und gerade des sozio-ökonomischen Impacts der Flutereignisse von 1342/43 wesentlich differenzierter geworden, weil über die narrative Überlieferung hinausgegangen wurde. Während die Zerstörung von Brücken tatsächlich stärker am Ober- und Mittellauf der großen mitteleuropäischen Ströme festzustellen war, hat gerade der Beleg von Überflutungen am Niederrhein gezeigt, wie verheerend auch dort die Lage in den Jahren 1342/43 war. Der Impact auf die Ernte konnte für sehr viel ausgedehntere Regionen plausibel gemacht werden, als es die süddeutschen Chroniken alleine nahelegen; die Vielzahl von Regulierungsmaßnahmen auf normativer Ebene und die rechtliche Adaption in Urkunden können zwar nicht mit letzter Sicherheit durch die Fluten erklärt werden, sprechen aber in ihrer Massivität doch eine klare Sprache. 
Zum zweiten haben sich Grenzen, aber auch neue Möglichkeiten naturwissenschaftlicher Proxydaten wie Baumringe gezeigt: Dass diese nur eine Saison abbilden und Extremereignisse schlecht widergeben, ist nicht neu. Und doch befinden sich, mit Ausnahme der fränkischen, alle lokalisierten Befunde aus Baumringen in grundsätzlicher Übereinstimmung mit den lokalen, anthropogenen Quellen. Aber gerade für die Jahre 1342/43 konnte anhand der schriftlichen Überlieferung dargestellt werden, welche Extreme sich in rascher Folge abwechselten: Eisgang, Winterhochwasser, Trockenheit, Stadtbrände, Starkregen, Julihochwasser und dann abrupt einsetzende Nahrungsmittelknappheit sowie möglicherweise ein Nachlassen der Bautätigkeit. Sowohl in meteorologischer und erst recht in sozio-ökonomischer Hinsicht handelt es sich bei den Hochwasserereignissen 1342/43 um einen compound event, der seine zerstörerische Wirkung nur in Kombination der genannten Ereignisse entfalten konnte. Im vorliegenden Beitrag ist gezeigt worden, dass sich über einen der consilience verpflichteten Zugang zu Extremereignissen wie den Hochwassern der Jahre 1342/43 eine Vielzahl von Faktoren identifizieren lassen, die erklären, warum der Starkregen von 1342 zu so ausgeprägter Erosion führen konnte und prononcierte, wenn auch nicht singuläre Spuren im Baumringbefund hinterließ. Auch die Feindatierung von Sedimentablagerungen lässt sich durch eine genauere Chronologie von Extremereignissen auf Schriftquellenbasis weiter präzisieren. Als Aufgabe bleibt bestehen, lokale Befunde aus den Schriftquellen mit den lokalen Baumfälldaten zu vergleichen, um ortsgebundene Baukonjunkturen nach den Flutzerstörungen zu identifizieren. Zum dritten stellt sich die Frage, ob die Bedeutung von Extremereignissen für die mittelalterliche Infrastrukturgeschichte so neue Konturen erhalten kann.

In keinem der untersuchten Fälle von Brückenneubauten haben wir es mit einem in sich geschlossenen Infrastruktursystem zu tun, das ausschließlich einen Hauptzweck verfolgte: nämlich den Hochwasserschutz zu priorisieren. Vielmehr erfüllten die adaptierten Brücken natürlich auch, ja sogar in erster Linie Verkehrs- und Repräsentationszwecke. Der Donaudurchstich und die niederrheinischen Deichbaugemeinschaften dienten sehr viel eindeutiger dem Hochwasserschutz, aber eben auch der herrschaftsmäßigen Durchdringung der Territorien durch die Landesherren. Erstaunlich ist dabei, dass sich diese politischen Intentionen aber erstmals in einem Aufgabenfeld trafen, das kostenaufwändig war und nur langsame Erfolge versprach. Dies waren bemerkenswerte erste Ansätze einer rein praktisch verstandenen "guten Herrschaft" durch kommunale und herrscherliche Autoritäten (bis hin zum Kaiser), die erstmals normativ-regulierend und infrastrukturell intervenierten, und das auf der Basis eines abgeleiteten, praktisch angewandten Umweltwissens. Dies geschah weder flächendeckend noch integrierend, das heißt durch Schaffung in- 
teragierender Normen, Institutionen und Infrastrukturen im Bereich der Nahrungsmittelvorsorge, die man nördlich der Alpen wohl erst nach den 1430er Jahren finden kann (Jörg 2008; Camenisch et al. 2016). Kommunale Organisation von Hochwasserschutz finden wir nördlich der Alpen schon früher, in der zweiten Hälfte des 14. Jahrhunderts und dann vor allem im 15. Jahrhundert (Schenk 2018) - trotzdem sind die Befunde aus den 1340er Jahren von großer Bedeutung, da sie zwar nicht die Kommunen, aber erstmals Landesherrn als (rudimentär greifbare) Akteure im Hochwasserschutz präsentieren. Interessant ist darüber hinaus, dass die Beispiele aus Niederbayern und vom Niederrhein keinen städtischen Ursprung haben - hier übernehmen ländlich geprägte Regionen eine Vorreiterrolle. An vielen Stellen konnte zumindest plausibel gemacht werden, dass regulierende Normen wie auch die punktuelle Anpassung von Infrastrukturen an die Hochwassererfahrung mit kurzer Verzögerung den Extremereignissen folgten, auch wenn eine direkte Kausalität - also eine explizite Bezugnahme auf das vorhergehende Extremereignis - fast nie nachzuweisen ist. Trotzdem scheint das Bild dicht genug, um von einer taktgebenden Funktion der Hochwasserereignisse 1342/43 für die Ausbildung von Infrastrukturen und Normen in der Mitte des 14. Jahrhunderts sprechen zu können. Hier wäre also die Hochwasserkrise tatsächlich die „punktuelle Umschlagssituation“ (Koselleck 1982: 617), die aber ohne eine (implizite) Selbstbeobachtung der Betroffenen (Schlögl 2016: 29; Meyer et al. 2013: 12) - conditio sine qua non eines an Luhmann angelehnten Umweltwissens zweiter Ordnung - nicht zu den konstatierten Adaptionsmaßnahmen führen konnte. Extremereignisse wie die Hochwasser von 1342/43 waren vielleicht weniger schlagartig, als wir bisher gedacht haben. Punktuell lässt sich auch eine direkte Beobachtung des Desasters, etwa durch die Boten des Herzogs von Geldern, feststellen. Doch meist erlaubt die Quellenlage solche Einblicke nicht, und der unmittelbare Peak eines Extremereignisses - die Zerstörung der Brücke, das Brechen der Dämme - war tatsächlich von kurzer Dauer. Erst die Verknappung des Nahrungsangebots ist ein Prozess, doch selbst dieser erfolgte im Fall der Extremniederschläge und der Hochwasser als rapid onset und kann daher nur schlecht mit feuchten Sommern, kalten Wintern und Dürren als slow onset-Ereignissen verglichen werden.

Eine (Erst-)Zumessung von künftiger Kritikalität der Infrastrukturen im Kontext meteorologischer Extremereignisse, hier verstanden als Umweltwissen zweiter Ordnung, also einer Reflexion der erfolgten Naturbeobachtung jenseits etablierter theologischer Deutungsmuster in einem technologisch-normativen Kontext, erfolgte also im diskutierten unmittelbaren Nachgang des Ereignisses - was eine (Neu-)Zumessung innerhalb der Lebenszeit einer bereits etablierten Infrastruktur, wie von Gerrit Schenk und Stefanie Eifert in ihrem Fallbeispiel diskutiert (Schenk \& Eifert 2018: 
65-72), eher ergänzt als widerlegt. Der Impact auf die Ernährungslage und die Wasserinfrastrukturen Mitteleuropas ist ebenfalls sehr viel deutlicher als zuvor, und doch wird auch klar, dass die Zeitgenossen im Rahmen ihrer Möglichkeiten energisch auf die natürlichen Extremereignisse reagierten, denen sie offensichtlich eine die Kritikalität von Infrastrukturen aufdeckende Wirkung zumaßen. Es scheint auch vertretbar - wenn auch im genannten „kontrolliert anachronistischen“ Sinn -, von einer Grundlegung vorstaatlicher Daseinsvorsorge (Vec 2008) im Nachgang der Jahre 1342/43 zu sprechen. Auch wenn die Systeme des Hochwasserschutzes durch Deiche und Durchstiche, der erhöhten Flutsicherheit von Brücken und der normativen Eingriffe in die Nahrungsmittelvorsorge fragmentarisch bleiben: In den 1340er Jahren finden wir so viele erste Belege für Aktivitäten einer Protodaseinsvorsorge (meist nur im Sinn der Sicherheit vor Hochwasser) durch Landesherren, dass es nicht völlig vermessen ist, die Hochwasserlagen der Jahre 1342/43 mit all ihren Begleiterscheinungen als äußerst folgenreiche Extremereignisse nicht nur in ihrem Schadenspotential zu betrachten. Die Wahrnehmung extremer Umweltfolgen führt so, verstanden als angewandtes und indirekt reflektiertes Umweltwissen, schon im Spätmittelalter zur Weiterentwicklung infrastrukturellen und normativen Wissens samt zugehöriger Praktiken.

\section{Danksagung}

Der Autor dankt an dieser Stelle herzlich für die Gelegenheit, das hier präsentierte Material im Jahr 2018 sowohl im Rahmen der Mittwochsvorträge des Leibniz-Instituts für die Geschichte und Kultur des östlichen Europa (GWZO) in Leipzig sowie, auf Einladung von Prof. Dr. Arnd Reitemeier, im Rahmen der Ringvorlesung Landesgeschichte an der GeorgAugust-Universität Göttingen vorzustellen. Die im Rahmen dieser Veranstaltungen erhaltenen Anregungen haben zur Entwicklung der These ebenso beigetragen wie die ausführlichen Rückmeldungen von Stephanie Eifert (Darmstadt). Besonderen Dank schulde ich Dr. Christian Popp und Jasmin Hoven-Hacker, M. A. (beide Germania Sacra, Göttingen) für ihre Hinweise auf die niederrheinische Überlieferung. Nicht zuletzt hat dieser Beitrag sehr von Gesprächen mit Kolleg*innen aus Geschichts- wie Geowissenschaften im Rahmen der Arbeitsgruppe „Climate Reconstruction and Impacts from the Archives of Societies" (CRIAS) im Rahmen des paläoklimatologischen Forschungsverbundes „Past Global Changes“ (PAGES) profitiert, wobei ausdrücklich Dr. Chantal Camenisch (Bern) und Dr. Andrea Kiss (Wien) zu nennen sind. 


\section{Anmerkungen}

1 Staatsarchiv Ludwigsburg, Bestand Reichsstadt Esslingen, B 169 Bü 643

\section{Literatur}

Alexandre, Pierre 1987. Le Climat en Europe au Moyen Age. Contribution à l'histoire des variations climatiques de 1000 à 1425, d'après les sources narratives de l'Europe occidentale. Paris: Éditions de l'EHESS.

Arnold, Werner 1980. DI 19. Die Inschriften der Stadt Göttingen. München: Druckenmüller: Nr. 5. URL: www.inschriften.net, urn:nbn:de:0238-di019g001k0000503 (10.01.2019)

Baethgen, Friedrich (Hg.) 1924. Johann von Winterthur, Chronik. Berlin: Weidmann.

Bahlke, Joachim et al. (Hg.) 1998. Handbuch der Historischen Stätten Böhmen und Mähren. Stuttgart: Kröner.

Bauch, Martin 2014. Die Magdalenenflut 1342 - ein unterschätztes Jahrtausendereignis? Mittelalter. Interdisziplinäre Forschung und Rezeptionsgeschichte (ISSN 2197-6120), 04. Februar 2014. URL: 〈http://mittelalter.hypotheses.org/3016〉

Bauch, Martin 2016. Nr. 1.3 Bronzekreuz vom Turm der St.-Albani-Kirche in Göttingen. In: Jiří Fajt und Markus Hörsch (Hg.). Ausstellungs-Katalog Karl IV. 1316-2016. Erste Bayerisch-Tschechische Landesausstellung. Prag, Wallenstein-Reitschule, 15.5.-25.9.2016; Prag, Carolinum, 14.5.-31.8.2016. Nürnberg, Germanisches Nationalmuseum, 20.10. 2016-5.3.2017. Praha: Narodní Galerie: 281.

Bauch, Martin 2017. Jammer und Not. Karl IV. und die natürlichen Rahmenbedingungen des 14. Jahrhunderts. Český Časopis Historický (115/4): 983-1016.

Bauch, Martin 2019. Consilience in der Vormoderne - Anmerkungen aus der Klimageschichte. NTM. Zeitschrift für Geschichte der Wissenschaften, Technik und Medizin (27/2): 185-192.

Bauer, Arnd W. 1993. Bodenerosion in den Waldgebieten des östlichen Taunus in historischer und heutiger Zeit - Ausmaß, Ursachen und geoökologische Auswirkungen. Frankfurt am Main: Institut für Physische Geographie.

Beckenbach, Elena et al. 2013. Spätmittelalterliche Starkregenereignisse und ihre geomorphologischen Kleinformen im Schönbuch (Süddeutschland): Erfassung mit hochauflösenden Fernerkundungsmethoden und sedimentologische Interpretation. Jahresberichte und Mitteilungen des Oberrheinischen Geologischen Vereins (95): 421-438.

Bell, Rainer 2007. Lokale und regionale Gefahren- und Risikoanalyse gravitativer Massenbewegungen an der Schwäbischen Alb. PhD thesis, Univ. Bonn. http://hss.ulb.uni-bonn. de/2007/1107/1107.htm

Bode, Georg (Hg.) 1905. Urkundenbuch der Stadt Goslar. Vierter Theil. Halle/S.: Hendel.

Böhmer, Johann Friedrich (Hg.) 1836. Codex diplomaticus moenofrancofurtanus, Vol. 1: [7941400]. Frankfurt /M.: Baer.

Böhmer, Johann Friedrich (Hg.) 1843. Michael de Leone, De chronicis temporum hominum modernorum. In: Johannes Victorienses und andere Geschichtsquellen Deutschlands im vierzehnten Jahrhundert. Stuttgart: Cotta: 466-479.

Borchard, Karl 1988. DI 27, Die Würzburger Inschriften bis 1525. Auf der Grundlage des Nachlasses von Theodor Kramer unter Mitarbeit von Franz Xaver Hermann. Wiesbaden: Reichert.

Bork, Hans-Rudolf 1988. Landschaftsentwicklung in Mitteleuropa. Wirkungen des Menschen auf Landschaften. Gotha \& Stuttgart: Klett-Perthes.

Bork, Hans-Jürgen und Jörg Bellstedt 2000. Mittel- und Jungholozäne Boden-, Substrat- und Reliefentwicklung im Bereich von Corvey. In: Hans-Georg Stephan (Hg.). Studien zur 
Siedlungsentwicklung und -struktur von Stadt und Reichskloster Corvey (800-1670). Eine Gesamtdarstellung auf der Grundlage archäologischer und historischer Quellen. Mit Beiträgen von Jörg Bellstedt u.a., Bd. 2. Neumünster: Wachholtz: 564-571.

Bork, Hans-Rudolf et al. 2011. Der 1000-jährige Niederschlag des Jahres 1342 und seine Folgen in Mitteleuropa. In: Falko Daim (Hg.). Strategien zum Überleben: Umweltkrisen und ihre Bewältigung. Mainz: Verlag des RGZM: 231-242.

Boček, Antonín (Hg.) 1860. Codex diplomaticus et epistolarius Moraviae. Bd. 7 (1334-1349). Olomouc: Skarnitz.

Brunner, Horst (Hg.) 1983. Das Hausbuch des Michael de Leone (Würzburger Liederhandschrift) der Universitätsbibliothek München ( $2^{\circ} \mathrm{Cod}$. Ms. 731). Göppingen: Kümmerle.

Büsching, Johann Gustav (Hg.) 1813. Jahrbücher der Stadt Breslau von Nicolaus Pol, Bd. 1. Breslau: Graß und Barth.

Bütow, Sascha 2015. Straßen im Fluss. Schiffahrt, Flussnutzung und der lange Wandel der Verkehrsinfrastruktur in der Mark Brandenburg und der Niederlausitz vom 13. bis zum 16. Jahrhundert. Berlin: Lukas-Verlag.

Camenisch, Chantal 2019. The Potential of Late Medieval and Early Modern Narrative Sources from the Area of Modern Switzerland for the Climate History of the Fourteenth Century. In: Martin Bauch und Gerrit J. Schenk (Hg.). The Crisis of the 14th Century: ,Teleconnections' between Environmental and Societal Change? Berlin: De Gruyter [im Druck].

Camenisch, Chantal et al. 2016. The 1430s: a cold period of extraordinary internal climate variability during the early Spörer Minimum with social and economic impacts in northwestern and central Europe. Climate of the Past (12): 2107-2126. https://doi.org/10. 5194/cp-12-2107-2016

Campbell, Bruce M.S. 2016. The Great Transition: Climate, Disease and Society in the LateMedieval World. Cambridge: Cambridge University Press.

Charpentier Ljungqvist, Frederik et al. 2018. Linking European building activity with plague history. Journal of Archaeological Science (98): 81-92.

Cihla, Michal und Michal Panáček 2011. Technological, Structural and Historical Aspects of the Gothic Bridge at Roudnice nad Labem. In: Bayerische Gesellschaft für Unterwasserarchäologie (Hg.) Archäologie der Brücken. Vorgeschichte, Antike, Mittelalter, Neuzeit. Regensburg: Pustet: 240-246.

Collet, Dominik und Maximilian Schuh (Hg.) 2017. Famines during the ,Little Ice Age'. Socionatural Entanglements in Premodern Societies. Cham: Springer.

Conrad, Klaus 1990. Pommersches Urkundenbuch. XI. Band 1341-1345. Köln \& Wien: Böhlau.

Cook, Edward R. et al. 2015. Old World megadroughts and pluvials during the Common Era. Science Advances (1/10): e1500561. https://doi.org/10.1126/sciadv.1500561

Deinhard, Johann Martin 1988. Lahnbrücke Weilburg. In: Bundesminister für Verkehr (Hg.) Steinbrücken in Deutschland. Düsseldorf: Beton-Verlag: 234-237.

Diehl, Adolf 1899. Urkundenbuch der Stadt Esslingen. Erster Band. Stuttgart: Kohlhammer.

Dotterweich, Markus et al. 2003. A 1: Mittel- und jungholozäne Siedlungsentwicklung, Landnutzung, Bodenbildung und Bodenerosion an einer mittelalterlichen Wüstung bei Friesen, Landkreis Kronach in Oberfranken. In: Hans-Rudolf Bork (Hg.). Bodenbildung, Bodenerosion und Reliefentwicklung im Mittel- und Jungholozän. Flensburg: Dt. Akad. f. Landeskunde: 17-56.

Dreibrodth, Stefan und Julian Wiethold 2015. Lake Belau and its catchment (Northern Germany): A key archive of environmental history in northern central Europe since the onset of agriculture. The Holocene (25/2): 296-322.

Ehmck, Diedrich R. und Wilhelm von Bippen 1876. Bremisches Urkundenbuch. Zweiter Band. Bremen: Müller.

Emler, Joseph (Hg.) 1892. Regesta diplomatica nec non epistolaria Bohemiae et Moraviae. Pars IV: 1333-1346. Praha: Greger.

Engels, Jens Ivo und Alfred Nordmann (Hg.) 2018. Was heißt Kritikalität? Zu einem Schlüsselbegriff der Debatte um Kritische Infrastrukturen. Bielefeld: Transcript.

Engels, Jens Ivo und Julia Obertreis 2007. Infrastrukturen in der Moderne. Einführung in ein junges Forschungsfeld. Saeculum (58/1): 1-12. 
Engels, Jens Ivo und Gerrit Jasper Schenk 2014. Macht der Infrastrukturen - Infrastrukturen der Macht. Überlegungen zu einem Forschungsfeld. In: Birte Förster und Martin Bauch (Hg.). Wasserinfrastrukturen und Macht. Politisch-soziale Dimensionen technischer Systeme von der Antike bis zur Gegenwart. München: Oldenbourg: 22-58.

Ficker, Julius (Hg.) 1851. Chronica episcoporum Monasteriensium. In: Die Münsterischen Chroniken des Mittelalters. Münster: Theissing: 1-91.

Förster, Birte und Martin Bauch (Hg.) 2015. Wasserinfrastrukturen und Macht. Politischsoziale Dimensionen technischer Systeme von der Antike bis zur Gegenwart. München: Oldenbourg.

Friese, Werner 1987. Telgter Urkundenbuch. Regesten zur Geschichte der Stadt Telgte und des Hauses Langen. Münster: Landschaftsverband Westfalen-Lippe.

Gauger, Maike 2010. Hochwasser und ihre Folgen - am Beispiel der Magdalenenflut 1342 in Hann. Münden. In: Bernd Hermann und Ulrike Kruse (Hg.). Schauplätze und Themen der Umweltgeschichte. Umwelthistorische Miszellen aus dem Graduiertenkolleg. Werkstattbericht. Göttingen: Universitätsverlag Göttingen: 95-106.

Gnädinger, Louise 1993. Johannes Tauler. Lebenswelt und mystische Lehre. München: Beck.

Gumbrecht, Hans-Ulrich 2013. Historisierung der Beobachtung zweiter Ordnung - eine epistemologische Rahmenerzählung. In: Perla Chinchilla Pawling, Aldo Mazzucchelli und Hans Ulrich Gumbrecht (Hg.). Beobachtung zweiter Ordnung im historischen Kontext. Niklas Luhmann in Amerika. Paderborn: Fink: 7-22.

Haas, Antonin 1954. Privilegia non regalium civitatum provincialium regni Bohemiae annorum 1232-1452. Privilegia nekrálovských měst českých $z$ let 1232-1452. Praha: Nakl. Českoslov. Akad. věd.

Herget, Jürgen et al. 2015. The millennium flood of July 1342 revisited. Catena (130): 82-94.

Herzog, Theo (Hg.) 1963. Landshuter Urkundenbuch. Neustadt/Aisch: Degener.

Hodenberg, Wilhelm von 1848. Hoyer Urkundenbuch. Fünfte Abtheilung: Archiv des Klosters Heiligenrode. Hannover: Jänecke.

Hoffmann, Hermann (Hg.) 1955. Würzburger Polizeisätze. Texte, Gebote und Ordnungen des Mittelalters 1125 bis 1495. Würzburg: Schöningh in Komm.

Hohensee, Ulrike et al. 2013. Monumenta Germaniae Historica. Constitutiones et acta publica imperatorum et regum. Zwölfter Band: Dokumente zur Geschichte des Deutschen Reichs und seiner Verfassung 1357-1359. Wiesbaden: Harrassowitz.

Höhlbaum, Konstantin 1882-86. Hansisches Urkundenbuch, Bd. 3: 1343-1360, hg. v. Verein für Hansische Geschichte. Halle /S.: Verlag der Buchhandlung des Waisenhauses.

Horrox, Rosemary (Hg.) 1994. The Black Death. Manchester \& New York: Manchester University Press.

Huber-Rebenich, Gerlinde et al. (Hg.) 2017. Wasser in der mittelalterlichen Kultur / Water in Medieval Culture. Gebrauch - Wahrnehmung - Symbolik / Uses, Perception, and Symbolism. Berlin: De Gruyter.

Hübner, Ignaz 1803. Merkwürdigkeiten der churbaierischen Hauptstadt Ingolstadt aus Urkunden zur Erleuchtung der vaterländischen Geschichte, Bd. 1. Ingolstadt: Attenkover.

Izdebski, Adam et al. 2016. Realising consilience: How better communication between archeologists, historians and natural scientists can transform the study of past climate change in the Mediterranean. Quaternary Science Reviews (136): 5-22.

Jacobeit, Jacundus, Andreas Philipp und Matthias Nonnenmacher 2006. Atmospheric circulation dynamics linked with prominent discharge events in Central Europe. Hydrology Sciences Journal (51/5): 946-965.

Jannsen, Wilhelm 1970. Ein niederrheinischer Fürstenhof um die Mitte des 14. Jahrhunderts. Rheinische Vierteljahrsblätter (34): 219-251.

Jarck, Hans-Heinrich 1982. Urkundenbuch des Klosters Osterholz: 1182-1651. Hildesheim: Lax.

Jörg, Christian 2008. Teure, Hunger, Großes Sterben. Hungersnöte und Versorgungskrisen in den Städten des Reiches während des 15. Jahrhunderts. Stuttgart: Hiersemann.

Kehr, Paul Fridolin (Hg.) 1899. Urkundenbuch des Hochstifts Merseburg, Bd. 1 (962-1357). Halle /S.: Hendel.

Kiss, Andrea 2009. Floods and weather in 1342 and 1343 in the Carpathian Basin. Journal of Environmental Geography (3-4/2): 37-47. 
Klein, Ulrich 2011. Archäologische Untersuchungen an der Weidenhäuser Brücke in Marburg/Lahn. In: Bayerische Gesellschaft für Unterwasserarchäologie (Hg.), Archäologie der Brücken. Vorgeschichte, Antike, Mittelalter, Neuzeit. Regensburg: Pustet: 264-270.

Knöpfler, Josef 1902. Die Reichsstädtesteuer in Schwaben, Elsaß und am Oberrhein zur Zeit Kaiser Ludwigs des Bayern. Zeitschrift für Württembergische Landesgeschichte (11): 287-351.

Kolbe, Günther 1988. Tauberbrücke Igersheim. In: Bundesminister für Verkehr (Hg.) Steinbrücken in Deutschland. Düsseldorf: Beton-Verl.: 42-44.

Kornrumpf, Gisela und Paul Gerhard Völker 1968. Die deutschen mittelalterlichen Handschriften der Universitätsbibliothek zu München. Wiesbaden: Harrassowitz.

Koselleck, Reinhart 1982. „Krise“. In: Otto Brunner, Werner Conze und Reinhart Koselleck (Hg.). Geschichtliche Grundbegriffe. Historisches Lexikon zur politisch-sozialen Sprache in Deutschland, Bd. 3: H-Me. Stuttgart: Klett-Cotta: 617-650.

Krüger, Sabine 1972. Krise der Zeit als Ursache der Pest? Der Traktat De mortalitate in Alamannia des Konrad von Megenberg. In: Festschrift für Hermann Heimpel zum 70. Geburtstag am 19. September 1971. Zweiter Band. Göttingen: Vandenhoeck \& Ruprecht: 839-883.

Laak, Dirk van 1999. Der Begriff „Infrastruktur“ und was er vor seiner Erfindung besagte. Archiv für Begriffsgeschichte (41): 280-299.

Labbé, Thomas und Gerrit Jasper Schenk (Hg.) 2018. Une histoire du sensible: La perception des victimes de catastrophe du XIIe au XVIIIe siècle. Actes du colloque international tenu à Lorsch (Allemagne, Hesse) du 11 au 14 décembre 2014. Eine Geschichte der Sensibilität: Die Wahrnehmung von Katastrophenopfern vom 12. bis zum 18. Jahrhundert. Tagungsband des internationalen Symposions, UNESCO Welterbe Kloster Lorsch, 11. bis 14. Dezember 2014. Turnhout: Brepols.

Leibniz, Gottfried Wilhelm (Hg.) 1711. De Origine et Abbatibus Monasterii Luccensis. In: Scriptores Rerum Brunsvicensium illustrationi inservientes. Bd. 3. Hannover: Förster: 690-699.

Leidinger, Georg (Hg.) 1918. Bayerische Chroniken des 14. Jahrhunderts. In: Monumenta Germaniae Historica. Scriptores rerum germanicarum. Hannover; Leipzig: Hahn: 151-175.

Liebscher, Frank und Joachim Schmiedel 1999. Spreebrücke Bautzen (Heilige-Geist-Brücke). In: Steinbrücken in Deutschland. Bd. 2: Berlin, Brandenburg, Mecklenburg-Vorpommern, Sachsen-Anhalt, Thüringen, hg. vom Bundesministerium für Verkehr, Bau- und Wohnungswesen. Erkrath: Beton-Verlag: 262-265.

Lorenz-Corswarem, Otto Graf von 1949. Zur Geschichte und Verwaltung der Koblenzer Moselbrücke. Jahrbuch für die Geschichte und Kultur des Mittelrheins und seiner Nachbargebiete (1): $15-28$.

Luff, Robert und Georg Steer (Hg.) 2003. Konrad von Megenberg, Das Buch der Natur. Bd. 2: Kritischer Text nach den Handschriften. Tübingen: Niemeyer.

McCormick, Michael 2011. History's Changing Climate: Climate Science, Genomics, and the Emerging Consilient Approach to Interdisciplinary History. Journal of Interdisciplinary History (42/2): 251-273.

Meier, Marietta 2016. Paradigmatische Fälle in der Geschichtswissenschaft: Kommentar zu einem historiographischen Normalfall. In: Ruben Marc Hackler und Katharina Kinzel (Hg.). Paradigmatische Fälle: Konstruktion, Narration und Verallgemeinerung von FallWissen in den Geistes- und Sozialwissenschaften. Basel: Schwabe: 157-166.

Menzel, Michael 2003. Regesten Kaiser Ludwigs des Bayern (1314-1347). Heft 7: Die Urkunden aus den Archiven und Bibliotheken Ober- und Niederbayerns. Köln \& Weimar \& Wien: Böhlau.

Meyer, Carla, Katja Patzel-Mattern und Gerrit Jasper Schenk 2013. Krisengeschichte(n). Eine Einleitung. In: Carla Meyer, Katja Patzl-Mattern und Gerrit Jasper Schenk (Hg.). Krisengeschichte(n). „Krise“ als Leitbegriff und Erzählmuster in kulturwissenschaftlicher Perspektive. Stuttgart: Steiner: 9-24.

Mindermann, Arend 2004. Urkundenbuch der Bischöfe und des Domkapitels von Verden, $B d$. 2: 1300-1380. Stade: Landschaftsverband der ehemaligen Herzogtümer Bremen und Verden. 
Monumenta Boica 1775. Monumenta boica. Volumen duodecim. München: Academia Scientiarum Maximilianea.

Moos, Peter von 1998. Das Öffentliche und das Private im Mittelalter. Für einen kontrollierten Anachronismus. In: Gert Melville und Peter von Moos (Hg.). Das Öffentliche und das Private in der Vormoderne. Köln \& Wien: Böhlau: 3-83.

Mühle, Eduard 2015. Breslau: Geschichte einer europäischen Metropole. Köln \& Weimar \& Wien: Böhlau.

Müller, Augustin 1929. Quellen- und Urkundenbuch des Bezirkes Teplitz-Schönau bis zum Jahre 1500. Prag: Selbstverlag des Vereins für die Geschichte der Deutschen in Böhmen.

Neueder, Hans 2012. Oberaltaich. Geschichte eines bedeutenden bayerischen Benediktinerklosters. Regensburg: Pustet.

Overmann, Alfred 1929. Urkundenbuch der Erfurter Stifter und Klöster. Teil 2: Die Urkunden der Stifter St. Marien und St. Severi (1331-1400). Magdeburg: Selbstverlag der Historischen Kommission.

Pfeiffer, Hans-Dieter und Joachim Schmiedel 1999. Elbebrücke Dresden (Augustusbrücke) In: Bundesministerium für Verkehr, Bau- und Wohnungswesen (Hg.) Steinbrücken in Deutschland. Bd. 2: Berlin, Brandenburg, Mecklenburg-Vorpommern, Sachsen-Anhalt, Thüringen. Erkrath: Beton-Verlag: 194-197.

Pfister, Christian 2005. Weeping in the Snow. The Second Period of Little Ice Age-Type Impacts. In: Wolfgang Behringer, Hartmut Lehmann und Christian Pfister (Hg.). Kulturelle Konsequenzen der „Kleinen Eiszeit“. Cultural Consequences of the „Little Ice Age“. Göttingen: Vandenhoeck \& Ruprecht: 31-86.

Pohlig, Matthias 2013. Vom Besonderen zum Allgemeinen? Die Fallstudie als geschichtstheoretisches Problem. Historische Zeitschrift (297): 297-319.

Posern-Klett, Karl Friedrich von (Hg.) 1868. Urkundenbuch der Stadt Leipzig. I. Band. Leipzig: Giesecke \& Devrient.

Riedel, Adolph Friedrich (Hg.) 1838-69. Codex Diplomaticus Brandenburgensis. Sammlung der Urkunden, Chroniken und sonstiger Quellenschriften für die Geschichte der Mark Brandenburg und ihrer Regenten. Berlin: Morin.

Rohr, Christian 2007. Extreme Naturereignisse im Ostalpenraum: Naturerfahrung im Spätmittelalter und am Beginn der Neuzeit. Köln \& Weimar \& Wien: Böhlau.

Rölker, Roland und Werner Delbanco 1997. Urkundenbuch des Stifts Börstel. Osnabrück: Selbstverlag des Vereins für Geschichte und Landeskunde von Osnabrück.

Ruland, Anton 1851. Die Würzburger Handschrift der k. Universitätsbibliothek zu München [CA]. Archiv des Historischen Vereins für Unterfranken und Aschaffenburg (11/2-3): $1-108$.

Sauer, Arno 1988. Moselbrücke Koblenz (Balduinbrücke). In: Bundesminister für Verkehr (Hg.), Steinbrücken in Deutschland. Düsseldorf: Beton-Verlag: 407-414.

Sauerland, Heinrich Volbert (Hg.) 1905. Urkunden und Regesten zur Geschichte der Rheinlande aus dem Vaticanischen Archiv. Bd. 3: 1342-1352. Bonn: Hanstein.

Schenk, Gerrit Jasper 2018. ,der straßen halb uff der Yll' Wasserwege im Elsass als ,kritische Infrastruktur' für Wirtschaft und Gesellschaft (1350-1550). In: Kurt Andermann und Nina Gallion (Hg.). Weg und Steg. Aspekte des Verkehrswesens von der Spätantike bis zum Ende des Alten Reiches. Ostfildern: Thorbecke: 121-152.

Schenk, Gerrit Jasper und Stefanie Eifert 2018. „Kritische Infrastrukturen“ als Ergebnisse individueller und kollektiver Kritikalitätszumessungen - ein Ansatz für die Mediävistik? In: Jens Ivo Engels und Alfred Nordmann (Hg.). Was heißt Kritikalität? Zu einem Schlüsselbegriff der Debatte um Kritische Infrastrukturen. Bielefeld: Transcript: 47-98.

Schilp, Thomas 2010. Essener Urkundenbuch. Regesten der Urkunden des Frauenstifts Essen im Mittelalter. Band I: Von der Gründung um 850 bis 1350. Düsseldorf: Droste.

Schlögl, Rudolf 2016. >Krise` als historische Form der gesellschaftlichen Selbstbeobachtung. Eine Einleitung. In: Rudolf Schlögl, Philipp R. Hoffmann-Rehnitz und Eva Wiebel (Hg.). Die Krise in der Frühen Neuzeit. Göttingen: Vandenhoeck \& Ruprecht: 9-32.

Schmidt, Berthold (Hg.) 1885. Urkundenbuch der Vögte von Weida, Gera und Plauen sowie ihrer Hausklöster Mildenfurth, Cronschwitz, Weida und zum Hl. Kreuz bei Saalburg. Erster Band: 1122-1356. Jena: Fischer.

Schmidt,Gustav 1878. Urkundenbuch der Stadt Halberstadt. Erster Theil. Halle /S.: Hendel. 
Schneider, Fedor (Hg.) 1910. Johann von Viktring, Liber certarum historiarum. Hannover \& Leipzig: Hahn.

Schneider, Georg 1949. Die Balduinbrücke über die Mosel in Koblenz. Jahrbuch für die Geschichte und Kultur des Mittelrheins und seiner Nachbargebiete (1): 1-14.

Schreg, Rainer 2013. Bodenerosion 1342 - ein Rechtsstreit in Esslingen. Archäologik. URL: http://archaeologik.blogspot.de/2013/01/bodenerosion-1342-ein-rechtsstreit-in.html (21.12.2018)

Schreiner, Johannes 1995. Der Schauzwang auf Deich und Schleuse „Die dwanck op den dijck ind sluese“. Die Entwicklung von Deichschauen am unteren Niederrhein vom Spätmittelalter bis zur Mitte des 17. Jahrhunderts. Bielefeld: Verlag für Regionalgeschichte.

Schreiner, Johannes 2001. Eine Idee gewinnt Gestalt: Die Entwicklung der Deichschauen an Maas und Waal, Rhein und Ijssel. In: Johannes Stinner und Karl-Heinz Tekath (Hg.). Gelre - Geldern - Gelderland. Geschichte und Kultur des Herzogtums Geldern. Geldern: Verlag des Historischen Vereins für Geldern und Umgegend: 269-278.

Schultheiss, Werner 1963. Urkundenbuch der Reichsstadt Windsheim von 741-1400. Würzburg: Schöningh.

Schweitzer, Caspar Anton (Hg.) 1853. Das Urkundenbuch des Abtes Andreas im Kloster Michelsberg bei Bamberg. Bamberg: Reindl.

Seils, Martin 2000. Holozäne Sediment- und Bodenverlagerungen im östlichen Harzvorland. Wirkungen und Ursachen nutzungsbedingter Landschaftsveränderungen. Halle: TriftVerlag.

Sigl, Michael et al. 2015. Timing and climate forcing of volcanic eruptions for the past 2,500 years. Nature (523): 543-549.

Sirocko, Frank (Hg.) 2009. Wetter, Klima, Menschheitsentwicklung: von der Eiszeit bis ins 21. Jahrhundert. Darmstadt: Wissenschaftliche Buchgesellschaft.

Sponheimer, Meinhard 1943. Urkundenbuch der Stadt Wetzlar. Zweiter Band: 1214-1350. Marburg: Elwert.

Standfuss, Friedrich 1988. Moselbrücke Trier (Römerbrücke). In: Bundesminister für Verkehr (Hg.), Steinbrücken in Deutschland. Düsseldorf: Beton-Verlag: 403-406.

Stephan, Hans-Georg 2013. Archäologische Untersuchungen zu mittelalterlichen Dorfwüstungen und Waldglashütten im Solling bei Bodenfelde im weiteren regionalen und gesamteuropäischen Kontext. Göttinger Jahrbuch (61): 325-354.

Stephan, Hans Georg 2011. Grabungen in mittelalterlichen Dorfwüstungen im Solling: Ergebnisse der Untersuchungen im Jahre 2010. Göttinger Jahrbuch (59): 207-217.

Symons, G[eorge] J[ames] (Hg.) 1891. Merle's Ms. Consideraciones temperiei pro 7 annis per Magistrum Willelmum Merle. The earliest known journal of weather kept by the Rev. William Merle, rector of Driby, Lincolnshire 1337-1344. London: E. Stanford.

Tadra, Ferdinand 1880. Cancellaria Arnesti. Formelbuch des ersten Prager Erzbischofs Arnest von Pardubic. Archiv für Österreichische Geschichte (61): 267-597.

Teschenmacher, Werner 1721. Annales Cliviae, Juliae, Montium, Marcae, Westphalicae, Rabensbergae, Geldriae et Zuphaniae. Frankfurt \& Leipzig: Nicolai.

Tetzlaff, Gerd et al. 2002. Das Jahrtausendhochwasser von 1342 am Main aus meteorologisch-hydrologischer Sicht [Meteorological and hydrological aspects of the 1000-year flooding event of the Main River in 1342]. Wasser $\mathcal{E}$ Boden (54/10): 41-49.

Vec, Miloš 2008. Daseinsvorsorge. In: Albrecht Cordes, Heiner Lück und Dieter Werkmüller (Hg.). Handwörterbuch zur Deutschen Rechtsgeschichte. Band 1: Aachen - Geistliche Bank. Berlin: Schmidt: 933-935.

Veesenmeyer, Gustav und Hugo Bazing 1898. Ulmisches Urkundenbuch, Bd. 2, Teil 1: Die Reichsstadt von 1315 bis 1356. Ulm: Kerler.

Verein für mecklenburgische Geschichte und Althertumskunde (Hg.) 1875. Mecklenburgisches Urkundenbuch, Vol. 9 (1337-1345). Schwerin: Stiller in Komm.

Vetter, Ferdinand (Hg.) 1910. Die Predigten Taulers aus der Engelberger und der Freiburger Handschrift sowie aus Schmidts Abschriften der ehemaligen Strassburger Handschriften. Berlin: Weidmann.

Vogtherr, Thomas 1988. Urkundenbuch der Stadt Uelzen. Hildesheim: Lax.

Wattenbach, Wilhelm (Hg.) 1851. Annales Mattseenses. In: Monumenta Germaniae Historica. Scriptores 9. Hannover: Hahn: 823-837. 
Wehking, Sabine 2006. DI 66. Die Inschriften des Landkreises Göttingen. Wiesbaden: Reichert: Nr. 9, URL: www.inschriften.net, urn:nbn:de:0238-di066g012k0000901 (10.01.2019)

Weigelt, Sylvia (Hg.) 2007. Johannes Rothe, Thüringische Landeschronik und Eisenacher Chronik. Berlin: Akademie-Verlag.

Weikinn, Curt 1958. Quellentexte zur Witterungsgeschichte Europas von der Zeitenwende bis zum Jahre 1850. Hydrographie, Teil 1 (Zeitenwende - 1500). Berlin: Akademie-Verlag.

White, Sam 2014. The Real Little Ice Age. Journal of Interdisciplinary History (44/3): 327-352.

Winkelmann, Eduard 1885. Acta imperii inedita, Bd. 2: Seculi XIII et XIV: Urkunden und Briefe zur Geschichte des Kaiserreichs und des Königreichs Sizilien in den Jahren 1200-1400. Innsbruck: Verlag der Wagner'schen Universitäts-Buchhandlung.

Wisselbach, Björn 2010. „Es führt über den Main... “ Frankfurts Alte Brücke. Gestern - heute - morgen. Frankfurt: Societäts-Verl.

Wyss, Arthur (Hg.) 1883. Die Limburger Chronik des Tilemann Elhen von Wolfhagen. Hannover: Hahn.

Zachová, Jana (Hg.) 1998. Chronicon Francisci Pragensis / Kronika Františka pražského. Praha: Nadace Patriae.

Zbinden, Eveline 2011. Das Magdalenen-Hochwasser von 1342 - der „hydrologische Gau“ in Mitteleuropa. Schweizer Wasserwirtschaftsverband: Wasser, Energie, Luft. Fachzeitschrift für Wasserwirtschaft (3): 193-203.

Zscheischler, Jakob et al. 2018. Future climate risk from compound events. Nature Climate Change (8): 469-477.

Zwierlein, Cornel 2011. Der gezähmte Prometheus: Feuer und Sicherheit zwischen Früher Neuzeit und Moderne. Göttingen: Vandenhoeck \& Ruprecht.

Šefců, Ondřej (Hg.) 2007. Karlův Most. Praha: Ottovo nakl.

Hinweis des Verlags Der Verlag bleibt in Hinblick auf geografische Zuordnungen und Gebietsbezeichnungen in veröffentlichten Karten und Institutsadressen neutral.

\section{Martin Bauch}

Freigeist Fellowship „The Dantean Anomaly“ (Volkswagen Stiftung), Abteilung Mensch und Umwelt

Leibniz-Institut für Geschichte und Kultur des östlichen Europa (GWZO) e.V.

Reichsstraße 4-6

04109 Leipzig

Deutschland

martin.bauch@uni-leipzig.de 